\title{
Mass scaling and nonadiabatic effects in photoassociation spectroscopy of ultracold strontium atoms
}

\author{
Mateusz Borkowski, ${ }^{1}$ Piotr Morzyński, ${ }^{1}$ Roman Ciuryło, ${ }^{1}$ Paul S. Julienne, ${ }^{2}$ Mi Yan, ${ }^{3}$ Brian J. DeSalvo, ${ }^{3}$ and T. C. Killian ${ }^{3}$ \\ ${ }^{1}$ Institute of Physics, Faculty of Physics, Astronomy and Informatics, Nicolaus Copernicus University, Grudziadzka 5, 87-100 Torun, Poland \\ ${ }^{2}$ Joint Quantum Institute, University of Maryland and National Institute of Standards and Technology, College Park, Maryland 20742, USA \\ ${ }^{3}$ Rice University, Department of Physics and Astronomy, Houston, Texas, 77251, USA
}

(Received 17 June 2014; published 16 September 2014)

\begin{abstract}
We report photoassociation spectroscopy of ultracold ${ }^{86} \mathrm{Sr}$ atoms near the intercombination line and provide theoretical models to describe the obtained bound-state energies. We show that using only the molecular states correlating with the ${ }^{1} S_{0}+{ }^{3} P_{1}$ asymptote is insufficient to provide a mass-scaled theoretical model that would reproduce the bound-state energies for all isotopes investigated to date: ${ }^{84} \mathrm{Sr},{ }^{86} \mathrm{Sr}$, and ${ }^{88} \mathrm{Sr}$. We attribute that to the recently discovered avoided crossing between the ${ }^{1} S_{0}+{ }^{3} P_{1} 0_{u}^{+}\left({ }^{3} \Pi_{u}\right)$ and ${ }^{1} S_{0}+{ }^{1} D_{2} 0_{u}^{+}\left({ }^{1} \Sigma_{u}^{+}\right)$potential curves at short range and we build a mass-scaled interaction model that quantitatively reproduces the available $0_{u}^{+}$ and $1_{u}$ bound-state energies for the three stable bosonic isotopes. We also provide isotope-specific two-channel models that incorporate the rotational (Coriolis) mixing between the $0_{u}^{+}$and $1_{u}$ curves which, while not mass scaled, are capable of quantitatively describing the vibrational splittings observed in experiment. We find that the use of state-of-the-art $a b$ initio potential curves significantly improves the quantitative description of the Coriolis mixing between the two $-8-\mathrm{GHz}$ bound states in ${ }^{88} \mathrm{Sr}$ over the previously used model potentials. We show that one of the recently reported energy levels in ${ }^{84} \mathrm{Sr}$ does not follow the long-range bound-state series and theorize on the possible causes. Finally, we give the Coriolis-mixing angles and linear Zeeman coefficients for all of the photoassociation lines. The long-range van der Waals coefficients $C_{6}\left(0_{u}^{+}\right)=3868(50)$ a.u. and $C_{6}\left(1_{u}\right)=4085(50)$ a.u. are reported.
\end{abstract}

DOI: 10.1103/PhysRevA.90.032713

PACS number(s): 34.50.Rk, 33.80.Be, 34.20.-b

\section{INTRODUCTION}

Photoassociation (PA) spectroscopy is a widely used tool for the study of atomic collisions and determination of bound-state energies of diatomic molecules [1]. In this process, two colliding cold atoms are bound together into an excited molecule by optical excitation. A recent focus for PA spectroscopy has been the study of molecules created by excitation to the red of an intercombination line $\left({ }^{1} S_{0} \rightarrow{ }^{3} P_{1}\right)$ in divalent atoms such as alkaline-earth metal atoms calcium [2] and strontium [3-5], and the rare-earth atom ytterbium [6-9]. The interest in intercombination line PA spectroscopy [10] is driven by its potential applications in the production of groundstate ultracold molecules [11], the potential for control of scattering lengths in ultracold collisions via optical Feshbach resonances [12], as well as coherent photoassociation $[13,14]$ and finally electron-proton mass ratio measurements [15].

The interactions between strontium atoms, both in their ground and excited states, have been extensively studied by means of intercombination line PA spectroscopy. Zelevinsky et al. [3] made the first observations with ${ }^{88} \mathrm{Sr}$ atoms confined in an optical lattice and recently Stellmer et al. [5] have reported similar measurements with ${ }^{84} \mathrm{Sr}$ atoms. Two-color photoassociation spectroscopy enabled Martinez de Escobar et al. [16] to accurately determine the scattering lengths of all $\mathrm{Sr}$ isotopes which helped to explain the low thermalization rate in ${ }^{88} \mathrm{Sr}$. In addition to the PA spectroscopy studies, both the interactions in the ground [17,18] and excited [19] states of the $\mathrm{Sr}_{2}$ molecule have been studied by Fourier transform spectroscopy.

The research into intercombination line PA is fueled by the possible use of optical Feshbach resonances (OFRs) [20-22] to enable optical control of the scattering lengths. This is especially important for ground-state strontium atoms because its ${ }^{1} S_{0}$ ground state and a lack of hyperfine structure in its bosonic isotopes precludes the existence of magnetic Feshbach resonances in this system. Early experiments with $\mathrm{Na}[23,24]$ and $\mathrm{Rb}[25,26]$ have shown that the usefulness of OFRs in alkali metals is greatly hindered by the large loss of atoms due to photoassociation. However, in the case of the narrow intercombination lines in divalent atoms such as $\mathrm{Ca}$, $\mathrm{Sr}$, and $\mathrm{Yb}$, these losses can be greatly reduced $[10,12]$ and useful changes in scattering lengths have been shown for both $\mathrm{Yb}$ [27] and recently Sr: a proof-of-concept investigation in a thermal gas [28] and an example of the use of OFRs as a means of controlling the collapse of a Sr Bose-Einstein condensate (BEC) [29].

All isotopes of $\mathrm{Sr}$ have been brought to quantum degeneracy. The most abundant isotope ${ }^{88} \mathrm{Sr}$ is known for its small negative scattering length $[16,30]$, which thwarted the early attempts $[31,32]$ at quantum degeneracy. The least abundant isotope, ${ }^{84} \mathrm{Sr}$, has excellent collision properties for evaporative cooling, so it was the first Bose-condensed isotope $[33,34]$. Since then, the isotope ${ }^{86} \mathrm{Sr}$ has also been condensed [35], while the thermalization problem in ${ }^{88} \mathrm{Sr}$ has been circumvented by sympathetic cooling with ${ }^{87} \mathrm{Sr}$ [36]. The narrow intercombination line enabled direct laser cooling of ${ }^{84} \mathrm{Sr}$ down to quantum degeneracy [37]. Degenerate Fermi [38] and Bose-Fermi gases [39] have also been reported. Strontium is being actively explored for its use in the making of ultracold molecules: ground-state strontium dimers $[11,40]$, and the heteronuclear $\mathrm{RbSr}$ molecules [41]. Rubidium-strontium mixtures have become especially promising after a degenerate quantum mixture of $\mathrm{Rb}$ and $\mathrm{Sr}$ atoms was obtained [42].

We report energy levels of the ${ }^{1} S_{0}+{ }^{3} P_{1} \mathrm{Sr}_{2}$ molecule obtained for the ${ }^{86} \mathrm{Sr}$ isotope, which complements the currently available data for ${ }^{88} \mathrm{Sr}$ [3] and ${ }^{84} \mathrm{Sr}$ [5], and we provide theoretical models of the interactions in the $\mathrm{Sr}_{2}$ molecule. A set of energy levels in the subradiant $1_{g}$ state of the strontium 
dimer has also become available [43], but is outside the scope of this article. The paper is organized as follows. In Sec. II, we briefly describe the experimental details and the PA data obtained for ${ }^{86} \mathrm{Sr}$. In Sec. III, we provide a theoretical model based on recent state-of-the-art ab initio potential curves [44] for the description of the long-range interactions in this excited state of the strontium dimer. We will use this model in Sec. IV to provide a quantitative description of vibrational splittings, as well as the nonadiabatic Coriolis effects and linear Zeeman coefficients [4] for all photoassociation lines reported to date. In the case of one of the isotopes, ${ }^{84} \mathrm{Sr}$, we will find a significant discrepancy between one of the experimental [5] and theoretical positions of one of the lines and theorize on its possible causes. In the case of ${ }^{88} \mathrm{Sr}$, we will show that the use of realistic potential curves significantly improves the quantitative description of the positions of strongly Coriolismixed energy levels over previous work [3]. We also show that the improvement of the description of Coriolis mixing is followed by better agreement of the respective Zeeman $g$ factors with the experimental data [4]. Finally, in Sec. VI we will investigate the mass scaling between strontium isotopes, that is, the possibility of using the same potential curves for the description of PA spectroscopy data for all isotopes. While mass scaling of only the long-range potentials was sufficient in the description of the energy levels near the ${ }^{1} S_{0}+{ }^{3} P_{1}$ asymptote in different isotopes of a similar species ytterbium [7], it fails in the case of strontium. We will explain this effect for the $0_{u}^{+}$series quantitatively by augmenting our model with the recently discovered $[19,44]$ curve crossing between the ${ }^{1} S_{0}+{ }^{3} P_{1}{ }^{3} \Pi_{u}$ curve (which supports the $0_{u}^{+}$series) and a ${ }^{1} \Sigma_{u}^{+}$curve correlating, remarkably, to the ${ }^{1} S_{0}+{ }^{1} D_{2}$ asymptote. Once mass scaling of the $0_{u}^{+}$bound states is achieved, we add a third channel representing the ${ }^{1} S_{0}+{ }^{3} P_{1} 1_{u}$ state. The final three-channel mass-scaled model reproduces the available $0_{u}^{+}$and $1_{u}$ bound state energies to within $0.5 \mathrm{MHz}$ on average.

TABLE I. Comparison of available experimental ${ }^{1} S_{0} \rightarrow{ }^{3} P_{1}$ photoassociation data with our long-range interaction model. The experimental data encompass all bosonic isotopes: ${ }^{88} \mathrm{Sr}$ (for total angular momentum $J=1$ [3], and recently $J=3$ [4]), ${ }^{84} \mathrm{Sr}$ data from Stellmer et al. [5], and our data for ${ }^{86} \mathrm{Sr}$. The numbers in parentheses denote the experimental uncertainty, where available. The model parameters were initially fitted to the bound-state energies for ${ }^{88} \mathrm{Sr}$ and then extended to other isotopes by only changing the respective short-range wave-function phase. For ${ }^{88} \mathrm{Sr}$ we also cite the theoretical bound-state energies from the original Zelevinsky et al. [3] model to show the improved description of Coriolis mixing between the two bound states at -8430 and $-8200 \mathrm{MHz}$ that our model provides. For ${ }^{84} \mathrm{Sr}$ we find it difficult to fit all of the energy levels together; therefore, the results shown are based on a fit that omits the $-1288-\mathrm{MHz}$ state. The observed drastic discrepancy between this state and the theoretical energy suggests that the $-1288 \mathrm{MHz}$ may not be a member of the $0_{u}^{+} J=1$ series. This is discussed in Sec. IV. We also give the Coriolis-mixing angles and Zeeman $g$ factors as measured in Ref. [4] and their theoretical counterparts as described in Sec. V.

\begin{tabular}{|c|c|c|c|c|c|c|c|c|c|c|}
\hline \multirow[b]{2}{*}{ Isotope } & \multirow[b]{2}{*}{ Series } & \multirow[b]{2}{*}{$J$} & \multicolumn{3}{|c|}{ Binding energy $E / h(\mathrm{MHz})$} & \multicolumn{2}{|c|}{ Mixing angle $\theta$} & \multicolumn{3}{|c|}{ Linear Zeeman coefficient $g$} \\
\hline & & & Expt. [3] & Theory [3] & Theory, this work & Theory [4] & This work & Expt. [4] & Theory [4] & Theory, this work \\
\hline \multirow[t]{9}{*}{${ }^{88} \mathrm{Sr}$} & $0_{u}^{+}$ & 1 & $-0.435(37)$ & -0.418 & -0.427 & $16.5^{\circ}$ & $17.46^{\circ}$ & $0.666(14)$ & 0.636 & 0.659 \\
\hline & $0_{u}^{+}$ & 1 & $-23.932(33)$ & -23.932 & -23.880 & $6.1^{\circ}$ & $6.07^{\circ}$ & $0.232(2)$ & 0.222 & 0.228 \\
\hline & $0_{u}^{+}$ & 1 & $-222.161(35)$ & -222.162 & -222.167 & $4.2^{\circ}$ & $4.04^{\circ}$ & $0.161(2)$ & 0.148 & 0.147 \\
\hline & $1_{u}^{u}$ & 1 & $-353.236(35)$ & -353.236 & -353.152 & $93.3^{\circ}$ & $93.89^{\circ}$ & $0.625(9)$ & 0.610 & 0.612 \\
\hline & $0_{u}^{+}$ & 1 & $-1084.093(33)$ & -1084.092 & -1084.022 & $3.8^{\circ}$ & $3.59^{\circ}$ & $0.142(2)$ & 0.128 & 0.131 \\
\hline & $1_{u}$ & 1 & $-2683.722(32)$ & -2683.723 & -2683.777 & $94.6^{\circ}$ & $94.90^{\circ}$ & $0.584(8)$ & 0.571 & 0.577 \\
\hline & $0_{u}^{+}$ & 1 & $-3463.280(33)$ & -3463.281 & -3463.346 & $5.1^{\circ}$ & $4.88^{\circ}$ & $0.193(3)$ & 0.174 & 0.173 \\
\hline & $1_{u}$ & 1 & $-8200.163(39)$ & -8112.699 & -8200.219 & $\left\{113.6^{\circ}, 175.9^{\circ}\right\}^{\mathrm{a}}$ & $112.58^{\circ}$ & $-0.149(2)$ & -0.592 & -0.024 \\
\hline & $0_{u}^{+}$ & 1 & $-8429.650(42)$ & -8420.133 & -8427.888 & $\left\{24.6^{\circ}, 84.9^{\circ}\right\}$ & $22.58^{\circ}$ & $0.931(13)$ & 1.333 & 0.774 \\
\hline Isotope & Series & $J$ & Expt. [4] & & Theory, this work & Theory [4] & This work & Expt. [4] & Theory [4] & Theory, this work \\
\hline \multirow[t]{2}{*}{${ }^{88} \mathrm{Sr}$} & $0_{u}^{+}$ & 3 & -0.63 & & -0.644 & $18.9^{\circ}$ & $18.92^{\circ}$ & $0.270(2)$ & 0.271 & 0.271 \\
\hline & $0_{u}^{+}$ & 3 & -132 & & -134.217 & $11.6^{\circ}$ & $12.22^{\circ}$ & $0.173(2)$ & 0.160 & 0.157 \\
\hline Isotope & Series & $J$ & Expt., this work & & Theory, this work & & This work & & & Theory, this work \\
\hline \multirow[t]{4}{*}{${ }^{86} \mathrm{Sr}$} & $0_{u}^{+}$ & 1 & $-1.633(10)$ & & -1.534 & & $12.93^{\circ}$ & & & 0.490 \\
\hline & $0_{u}^{+}$ & 1 & $-44.246(10)$ & & -43.850 & & $6.36^{\circ}$ & & & 0.214 \\
\hline & $1_{u}$ & 1 & $-159.984(50)$ & & -159.993 & & $97.31^{\circ}$ & & & 0.556 \\
\hline & $0_{u}^{+}$ & 1 & $-348.742(10)$ & & -348.825 & & $6.67^{\circ}$ & & & 0.206 \\
\hline Isotope & Series & $J$ & Expt. [5] & & Theory, this work & & This work & & & Theory, this work \\
\hline \multirow[t]{4}{*}{${ }^{84} \mathrm{Sr}$} & $0_{u}^{+}$ & 1 & $-0.320(10)$ & & -0.296 & & $19.56^{\circ}$ & & & 0.736 \\
\hline & $0_{u}^{+}$ & 1 & $-23.010(10)$ & & -23.011 & & $6.48^{\circ}$ & & & 0.242 \\
\hline & $0_{u}^{+}$ & 1 & $-228.380(10)$ & & -228.380 & & $3.47^{\circ}$ & & & 0.116 \\
\hline & $0_{u}^{+}$ & 1 & $-1288.290(10)$ & & -1144.492 & & $3.11^{\circ}$ & & & $0.113(0.101)^{b}$ \\
\hline
\end{tabular}

${ }^{\mathrm{a}}$ McGuyer et al. were uncertain which of the two $-8-\mathrm{GHz}$ states have $0_{u}^{+}$and $1_{u}$ symmetries, and therefore gave mixing angles for both of the possible assignments.

${ }^{\mathrm{b}}$ The number in parentheses denotes the $g$ factor calculated using a potential fitted to this state alone. 


\section{PHOTOASSOCIATION SPECTROSCOPY OF ${ }^{86} \mathrm{Sr}$}

To perform photoassociation (PA), we prepare ultracold ${ }^{86} \mathrm{Sr}$ atoms in an optical dipole trap (ODT) via laser cooling and trapping techniques similar to those used for other $\mathrm{Sr}$ isotopes $[45,46]$. The ODT is formed by the intersection of two mutually perpendicular beams focused to waists $\left(e^{-2}\right.$ intensity radii) of $100 \mu \mathrm{m}$. Both beams are generated from a 1064-nm, linearly polarized, multilongitudinal-mode fiber laser. A period of forced evaporation to a trap depth of $3.6 \mu \mathrm{K}$ yields $3 \times 10^{586} \mathrm{Sr}$ atoms at a temperature of $400 \mathrm{nK}$ and peak density of $10^{13} \mathrm{~cm}^{-3}$.

The PA beam is derived from a 689-nm master-slave diode laser system that has a linewidth of approximately $10 \mathrm{kHz}$. Short-term stability is provided by locking the laser frequency to a moderate finesse $(\mathcal{F}=2000)$ optical cavity, and long-term stability is assured through saturated absorption spectroscopy of the ${ }^{1} S_{0}-{ }^{3} P_{1}$ atomic transition in a vapor cell. The PA beam is red detuned with respect to the atomic transition using acousto-optic modulators (AOMs) and transported to the atoms through a single-mode optical fiber. In the interaction region, the beam is linearly polarized, with a waist of $700 \mu \mathrm{m}$ and peak intensity up to $50 \mathrm{~mW} / \mathrm{cm}^{2}$. During the application of the PA beam, to eliminate the ac Stark shift due to ODT beams, the ODT is modulated with a $50 \%$ duty cycle, a period of $462 \mu \mathrm{s}$, and a peak trap depth of $7.2 \mu \mathrm{K}$. The PA beam is applied when the ODT is off. Total PA time is varied from 16 to $830 \mathrm{~ms}$, depending upon the transition, to obtain a peak atom loss due to PA of approximately $50 \%$ with minimal change in sample temperature. The number of atoms and sample temperature are determined with time-of-flight absorption imaging using the ${ }^{1} S_{0}-{ }^{1} P_{1}$ transition at $461 \mathrm{~nm}$.

${ }^{86} \mathrm{Sr}$ photoassociation lines found in our experiment are listed in Table I. The binding energies $v_{0}$ were obtained by fitting the trap loss spectra with a realistic line-shape function [10]. The details of this procedure, the error budget, and the respective optical lengths are given in Appendix.

\section{LONG-RANGE INTERACTIONS}

In the original paper by Zelevinsky et al. [3], the energy levels obtained for ${ }^{88} \mathrm{Sr}$ were modeled using a five-channel model [10] which included both the relevant molecular states from the ${ }^{1} S_{0}+{ }^{3} P_{1}$ asymptote as well as ones from the ${ }^{1} S_{0}+{ }^{1} P_{1}$ and ${ }^{1} S_{0}+{ }^{3} P_{2}$ asymptotes. The energy levels for ${ }^{88} \mathrm{Sr}$ reproduced by this model are shown in Table I (labeled "Theory [3]").

In this paper, we will use a two-channel model based on the two long-range Hund's case (c) potential curves, $0_{u}^{+}$and $1_{u}$, that directly support the vibrational states near the ${ }^{1} S_{0}+{ }^{3} P_{1}$ asymptote. The molecular Hamiltonian can be partitioned in the following manner:

$$
H=T+V_{\text {int }}+V_{\text {rot }}
$$

Here, $T$ is the kinetic energy of the colliding atoms, $V_{\text {int }}$ covers the interaction potentials, while $V_{\text {rot }}$ is the rotational energy of the molecule. The kinetic energy term is diagonal regardless of chosen basis, with the diagonal term $-\left(\hbar^{2} / 2 \mu\right)\left(d^{2} / d R^{2}\right)$. In our homonuclear case, the reduced mass $\mu$ is equal to half the atomic mass of the chosen strontium isotope.

In this paper, we choose to work with potential curves based on state-of-the-art ab initio calculation of Skomorowski et al. [44] as opposed to using model potentials. Using the model in [47] we can write a two-channel Hund's case (c) atomic interaction Hamiltonian in terms of Hund's case (a) potential curves:

$$
V_{\mathrm{int}}=\left(\begin{array}{cc}
\mathcal{V}\left({ }^{3} \Pi_{u} ; R\right)-\frac{C_{3,0}}{R^{3}} & 0 \\
0 & \frac{1}{2}\left(\mathcal{V}\left({ }^{3} \Pi_{u} ; R\right)+\mathcal{V}\left({ }^{3} \Sigma_{u}^{+} ; R\right)\right)-\frac{C_{3,1}}{R^{3}}
\end{array}\right) .
$$

Our Hund case (a) potentials $\mathcal{V}\left({ }^{3} \Pi_{u}, R\right)$ and $\mathcal{V}\left({ }^{3} \Sigma_{u}^{+}, R\right)$ are based on the $a b$ initio potentials in Skomorowski et $\quad$ al.: $\quad \mathcal{V}\left({ }^{3} \Pi_{u}, R\right)=\left[V\left(\mathrm{c}^{3} \Pi_{u} ; R\right)-V\left(\mathrm{c}^{3} \Pi_{u} ; \infty\right)\right] \quad$ and $\mathcal{V}\left({ }^{3} \Sigma_{u}^{+}, R\right)=V\left(\mathrm{a}^{3} \Sigma_{u}^{+} ; R\right)-V\left(\mathrm{a}^{3} \Sigma_{u}^{+} ; \infty\right)$, where $V\left(\mathrm{c}^{3} \Pi_{u} ; R\right)$ and $V\left(\mathrm{a}^{3} \Sigma_{u}^{+} ; R\right)$ are the respective potential curves. The original ab initio potential curves were given in the convenient form of a short-range part combined with a Tang-Toennies damped [48] long-range part and enabled direct fitting of the potential parameters:

$$
\begin{aligned}
V(R)= & e^{-\alpha R-\gamma R^{2}}\left(A_{0}+A_{1} R+A_{2} R^{2}+A_{3} R^{3}+A_{4} R^{4}\right) \\
& -C_{12} f_{12} R^{-12}-C_{10} f_{10} R^{-10} \\
& -C_{8} f_{8} R^{-8}-C_{6} f_{6} R^{-6}
\end{aligned}
$$

where $f_{n}(R)$ is a Tang-Toennies damping function of the $n$th order [48]. During the fitting it was necessary to change the long-range $C_{6}$ and $C_{8}$ terms significantly. In order to retain the shape of the potential curves, we have refitted the remaining potential parameters to match the shape of the original $a b$ initio potentials, as shown in Fig. 1. The potential parameters used in our calculations are listed in Table II. Finally, we have included the resonant dipole interaction [49] into the model. In Skomorowski et al. this was achieved by spin-orbit mixing between states correlating to the ${ }^{1} S_{0}+{ }^{1} P_{1}$ and ${ }^{1} S_{0}+{ }^{3} P_{1}$ asymptotes. Since, however, we do not expect any new physics emerging from the inclusion of the far ${ }^{1} S_{0}+{ }^{1} P_{1}$ asymptote, we decide to model this mixing by simply adding the dipole terms artificially, following [47]. In this case, these terms are inversely proportional to the lifetime of the ${ }^{3} P_{1}$ atomic state in strontium:

$$
C_{3,0}=\frac{3}{2} \frac{\hbar}{\tau}\left(\frac{\lambda}{2 \pi}\right)^{3}
$$

and

$$
C_{3,1}=-C_{3,0} / 2 \text {. }
$$

The resonant dipole interaction is thus attractive in the $0_{u}^{+}$ curve while repulsive (and weaker) in the $1_{u}$ state. 


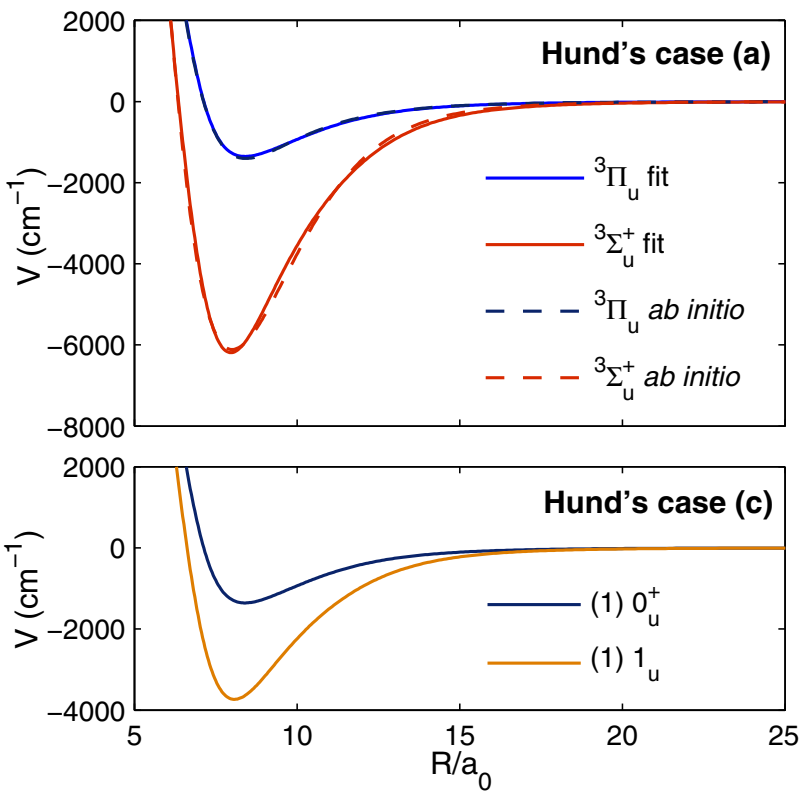

FIG. 1. (Color online) Potential curves correlating to the ${ }^{1} S_{0}+$ ${ }^{3} P_{1}$ asymptote used in the calculation of the theoretical binding energies. The dashed curves represent the original $a b$ initio Hund's case (a) potentials of Skomorowski et al. [44]. The solid lines are the potential curves fitted to the experimental data from photoassociation experiments. The top panel shows the potential curves in the Hund's case (a) representation, while the bottom panel shows them in the Hund's case (c) basis actually used in the calculation.

The remaining term in the molecular Hamiltonian is the rotational energy $V_{\text {rot }}$, which is diagonal in the Hund's case (e), but not in the Hund's case (c) representation. This causes

TABLE II. Potential parameters used in the calculation of theoretical ${ }^{1} S_{0}+{ }^{3} P_{1}$ bound-state energies in Table I. The potential parameters given here are to be used with Eq. (3). The only isotope-dependent parameter is $\alpha$, which is used to establish the correct short-range quantum defect for each curve.

\begin{tabular}{lrr}
\hline \hline Parameter & \multicolumn{1}{c}{${ }^{3} \Sigma_{u}^{+}$} & \multicolumn{1}{c}{${ }^{3} \Pi_{u}$} \\
\hline$A_{0}$ & $1.29406314 \times 10^{2}$ & $5.78723038 \times 10^{6}$ \\
$A_{1}$ & $-7.90551852 \times 10^{1}$ & $-3.46113235 \times 10^{6}$ \\
$A_{2}$ & $1.87863441 \times 10^{1}$ & $7.79019763 \times 10^{5}$ \\
$A_{3}$ & $-1.96979418 \times 10^{0}$ & $-7.85317879 \times 10^{4}$ \\
$A_{4}$ & $7.88636443 \times 10^{-2}$ & $3.01833743 \times 10^{3}$ \\
$\gamma$ & $7.61382806 \times 10^{-2}$ & $1.34967817 \times 10^{-3}$ \\
$\beta$ & 1.00 & 1.03238202 \\
$\alpha\left({ }^{88} \mathrm{Sr}\right)$ & 0.045647282 & 1.99168225 \\
$\alpha\left({ }^{86} \mathrm{Sr}\right)$ & 0.045690735 & 1.99188286 \\
$\alpha\left({ }^{84} \mathrm{Sr}\right)$ & 0.045301189 & 1.99037413 \\
$C_{12}$ & $-5.31841848 \times 10^{9}$ & $-1.06415514 \times 10^{10}$ \\
$C_{10}$ & $2.20495 \times 10^{8}$ & $5.24064 \times 10^{7}$ \\
$C_{8}$ & $2.3574797 \times 10^{6}$ & $3.4156471 \times 10^{5}$ \\
$C_{6}$ & $4.3015063 \times 10^{3}$ & $3.8683912 \times 10^{3}$ \\
$C_{3,0}$ & $1.52356615 \times 10^{-2}$ \\
\hline \hline
\end{tabular}

rotational (Coriolis) mixing between the two molecular states:

$$
V_{\mathrm{rot}}=B(R)\left(\begin{array}{cc}
J(J+1)+2 & -\sqrt{4 J(J+1)} \\
-\sqrt{4 J(J+1)} & J(J+1)
\end{array}\right)
$$

with $B(R)=\hbar^{2} / 2 \mu R^{2}$. In a similar study with ytterbium atoms [7], the large dispersion between the two potentials caused by the differences in the resonant dipole interaction [see Eq. (5)] made it possible to forego Coriolis mixing and fit the available energy level data with a single-channel potential. Here, however, the dipole interaction is much weaker, making it necessary to include this mixing in order to properly recreate bound-state energies very close to the dissociation limit. Not including the Coriolis-mixing limits the accuracy of the model to about $1 \mathrm{MHz}$ for most energy levels. The exception to this rule is the case of strongly mixed states, like the $-8200 \mathrm{MHz}$ and $-8430 \mathrm{MHz}$ states in ${ }^{88} \mathrm{Sr}$, where this inaccuracy is drastic, as shown in Sec. IV.

Theoretical bound-state energies can be obtained by solving the coupled-channel Schrödinger equation $H \Psi=E \Psi$, where $\Psi=\left[\Psi\left(0_{u}^{+} ; R\right), \Psi\left(1_{u} ; R\right)\right]^{T}$ is the two-channel wave function. We solve these equations numerically using the matrix DVR method [50] with nonlinear coordinate scaling.

The long-range parameters of the Hund's case (a) potentials $V\left(\mathrm{a}^{3} \Sigma_{u}^{+} ; R\right)$ and $V\left(\mathrm{c}^{3} \Pi_{u} ; R\right)$ were fitted to the experimental data using the nonlinear least-squares method. The long-range resonant dipole $C_{3}$, the two van der Waals terms $C_{6}\left(\mathrm{a}^{3} \Sigma_{u}^{+}\right)$and $C_{6}\left(\mathrm{c}^{3} \Pi_{u}\right)$, and the two respective $C_{8}$ terms were first used to match the vibrational energies only for the $J=1{ }^{88} \mathrm{Sr}$ data. To a good approximation, the $C_{3}$ and $C_{6}$ (and to a lesser extent $C_{8}$ ) coefficients determine the vibrational splittings. Here, the short-range $\alpha$ terms can be used to tune the phases (or "quantum defects") of the short-range parts of the radial wave functions and effectively shift the whole vibrational series in place. This parameter was chosen for phase adjustments in an attempt to preserve the shape of the short-range potential as much as possible. The resulting bound-state energies can be seen in Table I. The energy levels for $J=3$ were computed using the same set of parameters.

The vibrational level data for the remaining two isotopes ${ }^{86} \mathrm{Sr}$ and ${ }^{84} \mathrm{Sr}$ was modeled by adjusting only the two $\alpha$ coefficients on a per-isotope basis in order to fix the right short-range wave-function phase. The differences between the $\alpha$ coefficients for different isotopes do not exceed $1 \%$. Therefore, the theoretical bound-state energies listed in the "Theory (this work)" of Table I were calculated using slightly different potential curves that shared the same set of the van der Waals $C_{6}$ and $C_{8}$ coefficients: $C_{6}\left({ }^{3} \Sigma_{u}^{+}\right) \approx 4302$ a.u. and $C_{6}\left({ }^{3} \Pi_{u}\right) \approx 3868$ a.u., which correspond to $C_{6}\left(0_{u}^{+}\right)=$ 3868 a.u. and $C_{6}\left(1_{u}\right)=4085$ a.u. in the Hund's case (c) representation. The $C_{8}$ coefficients are $C_{8}\left({ }^{3} \Sigma_{u}^{+}\right) \approx 2.36 \times 10^{6}$ a.u. and $C_{8}\left({ }^{3} \Pi_{u}\right) \approx 3.42 \times 10^{5}$ a.u. We tentatively assign an error bound of 50 a.u. to each of the Hund's case (c) $C_{6}$ coefficients: a change of this size introduces a change in the vibrational splittings that can not be compensated using the other long-range parameters. We choose to assign uncertainties to the Hund's case (c) parameters because those directly affect the positions of the photoassociation resonances. In the Hund's case (a) representation, this results in uncertainties of 50 and 150 a.u. for $C_{6}\left({ }^{3} \Pi_{u}\right)$ and $C_{6}\left({ }^{3} \Sigma_{u}^{+}\right)$assuming our 
TABLE III. Van der Waals coefficients $C_{6}$ for $\mathrm{Sr}_{2}$ molecular states correlating to the ${ }^{1} S_{0}+{ }^{3} P_{1}$ asymptote given in Hund's case (a) and (c) bases.

\begin{tabular}{lcccc}
\hline \hline Source & $C_{6}\left({ }^{3} \Pi_{u}\right)$ & $C_{6}\left({ }^{3} \Sigma_{u}^{+}\right)$ & $C_{6}\left(0_{u}^{+}\right)$ & $C_{6}\left(1_{u}\right)$ \\
\hline $\begin{array}{l}\text { Empirical [3] } \\
\text { Ab initio [51] }\end{array}$ & $3513 \pm 200^{\mathrm{a}}$ & $4035 \pm 600^{\mathrm{a}}$ & $3513 \pm 200$ & $3774 \pm 200$ \\
$\begin{array}{l}\text { Ab initio } \\
\text { (pure) [52] }\end{array}$ & 3951 & 4488 & $3951^{\mathrm{b}}$ & $4220^{\mathrm{b}}$ \\
$\begin{array}{l}\text { A } \text { initio } \\
\text { (recomm.) [52] }\end{array}$ & $3771 \pm 32^{\mathrm{a}}$ & $4231 \pm 98^{\mathrm{a}}$ & $3771 \pm 32$ & $4001 \pm 33$ \\
This work & $3868 \pm 50$ & $4302 \pm 150$ & $3868 \pm 50$ & $4085 \pm 50$ \\
\hline \hline
\end{tabular}

${ }^{\mathrm{a} C a l c u l a t e d}$ via $C_{6}\left({ }^{3} \Pi_{u}\right)=C_{6}\left(0_{u}^{+}\right)$and $C_{6}\left({ }^{3} \Sigma_{u}^{+}\right)=2 C_{6}\left(1_{u}\right)-$ $C_{6}\left(0_{u}^{+}\right)$[47] from Hund's case (c) values.

${ }^{\mathrm{b} C a l c u l a t e d}$ via $C_{6}\left(0_{u}^{+}\right)=C_{6}\left({ }^{3} \Pi_{u}\right)$ and $C_{6}\left(1_{u}\right)=\left[C_{6}\left({ }^{3} \Sigma_{u}^{+}\right)-\right.$ $\left.C_{6}\left({ }^{3} \Pi_{u}\right)\right] / 2$ [47] from Hund's case (a) values.

${ }^{\mathrm{c}}$ Reference [51] did not give error bounds for the calculated values.

estimations should be treated as maximum errors. Our fitted resonant dipole term $C_{3,0} \approx 0.01524$ a.u. which corresponds to a natural linewidth of the atomic ${ }^{3} P_{1}$ state of $1 / \tau=$ $2 \pi \times 7501.9 \mathrm{~Hz}$. Our lifetime of $21.215 \mu$ s agrees well with both the theoretical determination [44] of $21.4 \mu \mathrm{s}$ and, not surprisingly, the empirical value of $21.5 \mu$ s from the previous photoassociation experiment [3].

Our $C_{6}$ coefficients can be compared to previous works (see Table III). The first photoassociation-based determination [3] gives $C_{6}\left(0_{u}^{+}\right)=3513$ a.u. and $C_{6}\left(1_{u}\right)=3774$ a.u., respectively with an estimated error bound of 200 a.u. These values appear to be underestimated when compared to both our work and the recent $a b$ initio determinations. The Hund's case (a) $C_{6}$ values originally calculated by Mitroy and Zhang [51] and used in the model in Skomorowski et al. are 4488 and 3951 a.u. for the respective ${ }^{3} \Sigma_{u}^{+}$and ${ }^{3} \Pi_{u}$ states, which is about one and a half error bound larger than our empirical determination. We note, however, that no error bound was given for their calculation. A new $a b$ initio calculation by Safronova et al. [52] is also available. The first set of coefficients $C_{6}\left(0_{u}^{+}\right)=3821$ a.u. and $C_{6}\left(1_{u}\right)=4055$ a.u. is of pure $a b$ initio origin (labeled "Ab initio (pure) [52]" in Table III) and fits our data perfectly. The second set of coefficients [" $A b$ initio (recomm.) [52]"] $C_{6}\left(0_{u}^{+}\right)=3771(32)$ a.u. and $C_{6}\left(1_{u}\right)=$ 4001(33) a.u., includes several empirical corrections [53] and while the difference is larger, the data still agree with ours to within mutual error bars. It should be noted that the currently available $\mathrm{Sr}$ photoassociation data is only weakly sensitive to the $C_{8}$ values. Therefore, $C_{8}\left({ }^{3} \Sigma_{u}^{+}\right)$and $C_{8}\left({ }^{3} \Pi_{u}\right)$ should only be considered as potential fitting parameters and not used for comparison with other determinations. Not surprisingly, they do not agree with the newest available $a b$ initio calculations [52].

\section{LINE POSITIONS}

The agreement between our two-channel model and the experimentally determined ${ }^{88} \mathrm{Sr}$ bound-state energies is excellent. The theoretical bound-state energies match the experimental line positions to within the error bars with the exception of the two states at $-8430 \mathrm{MHz}\left(0_{u}^{+}\right)$and $-8200 \mathrm{MHz}\left(1_{u}\right)$,

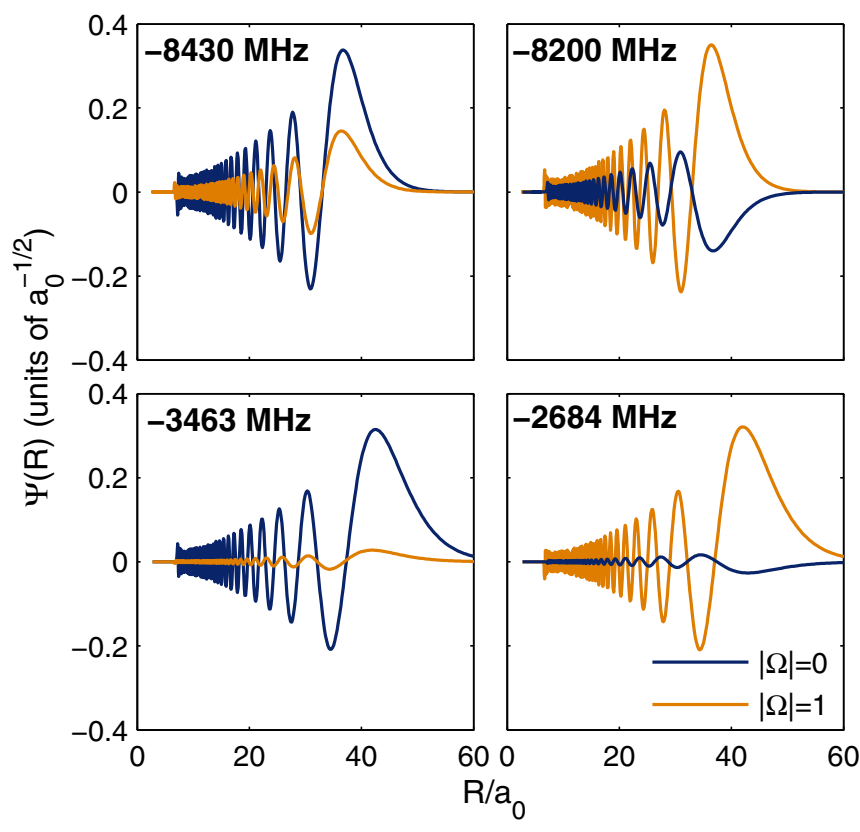

FIG. 2. (Color online) Coriolis mixing between the $0_{u}^{+}$and $1_{u}$ series. The upper two plots show the two-channel wave functions of the strongly mixed ${ }^{88} \mathrm{Sr} J=1$ levels at -8430 and $-8200 \mathrm{MHz}$ $\left(\theta=24^{\circ}\right.$ and $114^{\circ}$, respectively). In the case of the $-8430-\mathrm{MHz}$ level, the $0_{u}^{+}$component (dark blue lines) constitutes the majority of the two-channel wave function, while the $-8200-\mathrm{MHz}$ state is predominantly of the $1_{u}$ symmetry (orange lines). For comparison, in the lower part of the figure we show two relatively pure $\left(\theta=5^{\circ}\right.$ and $\left.95^{\circ}\right) 0_{u}^{+}$and $1_{u}$ states at -3463 and $-2684 \mathrm{MHz}$, respectively.

where the accuracy is limited to about $2 \mathrm{MHz}$; see Table I. These two bound states are very strongly mixed by the Coriolis coupling, partially due to the large wave-function overlap. The two-channel wave functions for these two energy levels are shown in Fig. 2. Our theoretical model predicts mixing angles, as defined in Sec. $\mathrm{V}$, of about $\theta \sim 23^{\circ}$, which is in good agreement with the recent empirical determination based on Zeeman shifts of photoassociation lines [4]. Compared to the other energy levels, quantitative description of these two states is very difficult because the Coriolis splitting between them is strongly dependent on the relative phases of their respective wave functions which in turn are determined by the relatively unknown short-range parts of their supporting potential curves. We note that our use of realistic ab initio potentials improved the agreement dramatically: the previous model [3] was off by several tens of $\mathrm{MHz}$, while ours reduces that down to less than $2 \mathrm{MHz}$. For completeness, we also show the theoretical counterparts of the two $J=3$ energy levels reported recently in [4].

The same long-range model (except for the adjustment of $C_{8}$ terms) applied to the case of ${ }^{86} \mathrm{Sr}$ yields a slightly worse fit, with inaccuracies reaching up to $0.5 \mathrm{MHz}$, which can be attributed to the still imperfect description of Coriolis mixing (in the case of the top bound state) or to the impact of shortrange curve crossings on the vibrational splittings as discussed in detail in Sec. VI C.

The case of ${ }^{84} \mathrm{Sr}$ atoms has been experimentally investigated recently by Stellmer et al. [5]. In this analysis we, however, leave out the $1_{u}$ state at $-351 \mathrm{MHz}$ state reported in [5], but not 
in the energy level table in a subsequent review paper [54,55]. Only bound states of $0_{u}^{+}$symmetry are therefore available for the case of ${ }^{84} \mathrm{Sr}$ atoms.

The experimental bound-state energies for the ${ }^{84} \mathrm{Sr} 0_{u}^{+}$ symmetry obtained by Stellmer et al. [5] have vibrational spacings that can not be fully reproduced by our long-range model. Given the excellent agreement between theory and experiment in the case of the two other isotopes we can safely assume that at least the two van der Waals parameters $C_{6}\left({ }^{3} \Pi_{u}\right)$ and $C_{6}\left({ }^{3} \Sigma_{u}^{+}\right)$and the resonant dipole term $C_{3}$ of the two potentials are correct. Similarly to the case of ${ }^{86} \mathrm{Sr}$, we have adjusted the two $\alpha$ terms to fit the model to the ${ }^{84} \mathrm{Sr}$ data. Due to the lack of available $1_{u}$ bound-state data, the $\alpha\left({ }^{3} \Sigma_{u}^{+}\right)$ parameter was fitted to provide the best fit of $0_{u}^{+}$energy levels. If we fit the model to all of the $0_{u}^{+}$bound states except for the $-1288 \mathrm{MHz}$ level, we obtain a fit to better than $25 \mathrm{kHz}$ for all but the $-1288-\mathrm{MHz}$ state. This is shown in Table I. The latter state appears not to follow the $J=10_{u}^{+}$series, as the closest energy level predicted by our long-range model is located at about $-1144 \mathrm{MHz}$, that is, almost $150 \mathrm{MHz}$ away. On the other hand, if we instead fit our $0_{u}^{+}$series to the $-1288-\mathrm{MHz}$ state, we obtain a model that is in drastic disagreement with the remaining experimental bound-state positions giving line positions of $-0.57,-29.3$, and $-269.3 \mathrm{MHz}$.

Our long-range model's inability to describe the ${ }^{84} \mathrm{Sr}$ vibrational spectrum suggests that the $-1288-\mathrm{MHz}$ state could be either perturbed by an adjacent state in a different potential curve or it could have been mislabeled as a $J=10_{u}^{+}$state. In fact, given that the experiment [5] was performed in a BoseEinstein condensate, it is plausible that this photoassociation line is supported by one of the subradiant gerade states $\left(0_{g}^{+}\right.$ or $1_{g}$ ), much like those observed in ytterbium [9]. In this case, it would be entirely unperturbed by the $0_{u}^{+}$and $1_{u}$ series due to symmetry. This theory could be confirmed by actually finding an unperturbed $0_{u}^{+}$state near $-1144 \mathrm{MHz}$ as predicted by the long-range model. It is important to note that not finding such state does not necessarily disprove its existence; the intensity of a PA line can be greatly diminished if the ground-state wave function has a node at the Condon point for such transition $[9,56]$.

We have verified that the apparent shift of the $-1288-\mathrm{MHz}$ state is not caused by a simple rotational state labeling error. In such scenario, this energy level could indeed have $J=$ 1 , but the remaining three $0_{u}^{+}$states could have $J=3$ and therefore lay closer to the dissociation limit. However, if this were the case, the theoretical bound-state energies would be -159 and $-1.5 \mathrm{MHz}$ for $J=3$ with the most weakly bound state disappearing altogether.

We have tested a possibility that the $-1288-\mathrm{MHz}$ state belongs to the $1_{u}$ symmetry, which is plausible as so far no $1_{u}$ resonances were found in ${ }^{84} \mathrm{Sr}$. However, if we fit the $\alpha\left({ }^{3} \Sigma_{u}^{+}\right)$parameter so that our model reproduces a $1_{u}$ state at $-1288 \mathrm{MHz}$, another bound state of this symmetry emerges at $-2.90 \mathrm{MHz}$. Its presence causes a Coriolis shift of the top weakly bound $0_{u}^{+}$state to $-0.21 \mathrm{MHz}$, that is, over 10 error bounds away from the experimental value of $-0.320(10) \mathrm{MHz}$. Therefore, we view such possibility as unlikely.

Finally, the $0_{u}^{+}$vibrational spacings could be influenced by the strong short-range spin-orbit mixing with other electronic states. For example, the original Mies et al. model [47] contains nondiagonal spin-orbit terms between $1_{u}$ curves correlating to the ${ }^{1} S_{0}+{ }^{3} P_{1}$ and ${ }^{1} S_{0}+{ }^{3} P_{2}$ asymptotes. Such strong mixing could create very wide resonances spanning several bound states near the ${ }^{1} S_{0}+{ }^{3} P_{1}$ dissociation limit. Such a case could occur in all PA spectroscopy experiments involving divalent atoms such as ytterbium or calcium, but to the best of our knowledge, no empirical evidence has so far been found. The impact of this mixing can be somewhat diminished by the fact that potential curves of different $j$ states of the same ${ }^{3} P$ asymptote are largely parallel and do not cross. In strontium, however, the situation is further complicated by an additional ${ }^{1} S_{0}+{ }^{1} D_{2} 0_{u}^{+}$curve crossing the $0_{u}^{+}$state probed in photoassociation experiment. We will explore its consequences in Sec. VI.

\section{CORIOLIS MIXING AND ZEEMAN SPLITTINGS}

The Coriolis terms in the rotational Hamiltonian cause nonadiabatic mixing between the $0_{u}^{+}$and $1_{u}$ components of the molecular wave function. This effect can be quantified by introducing a mixing angle $\theta$ defined by writing the total molecular wave function as

$$
\Psi(R)=\left[\cos \theta \tilde{\Psi}\left(0_{u}^{+} ; R\right), \sin \theta \tilde{\Psi}\left(1_{u} ; R\right)\right]^{T} .
$$

Here, we define the two reference functions $\tilde{\Psi}\left(0_{u}^{+} ; R\right)$ and $\tilde{\Psi}\left(1_{u} ; R\right)$, which are the two wave-function components normalized separately via

$$
\tilde{\Psi}\left(0_{u}^{+} ; R\right)=\Psi\left(0_{u}^{+} ; R\right)\left(\int_{0}^{\infty} \Psi\left(0_{u}^{+} ; R\right)^{2} d R\right)^{-1 / 2}
$$

and

$$
\begin{aligned}
\tilde{\Psi}\left(1_{u} ; R\right)= & \Psi\left(1_{u} ; R\right)\left(\int_{0}^{\infty} \Psi\left(1_{u} ; R\right)^{2} d R\right)^{-1 / 2} \\
& \times \operatorname{sgn}\left(\int_{0}^{\infty} \Psi\left(0_{u}^{+} ; R\right) \Psi\left(1_{u} ; R\right) d R\right) .
\end{aligned}
$$

The signum function above ensures that our phase convention is compatible with the one in Ref. [4]. It is straightforward to verify that with these definitions Eq. (7) yields a correctly normalized two-channel wave function. Finally, we note that the transformation $\theta \rightarrow \theta+180^{\circ}$ only changes the sign of the total wave function without altering its internal phase relationship. We therefore decide on having $0^{\circ} \leqslant \theta<180^{\circ}$, again in accordance with [4]. In this convention, a pure $0_{u}^{+}$ state has $\theta=0^{\circ}$ or $\theta \rightarrow 180^{\circ}$, while $\theta=90^{\circ}$ for a pure $1_{u}$ state.

Table I lists our Coriolis-mixing angles $\theta$ for each of the considered energy levels. For completeness, we also include mixing angles from Ref. [4] which are in good agreement with ours. For two of the most deeply bound states in ${ }^{88} \mathrm{Sr}$ at -8200 and $-8430 \mathrm{MHz}$, McGuyer et al. give two different mixing angles as their theoretical model was not accurate enough to ascertain which of these two energy levels belong to the $0_{u}^{+}$ and $1_{u}$ series. In our model, however, the $-8200-\mathrm{MHz}$ line clearly belongs to the $1_{u}$ series, while the $-8430 \mathrm{MHz}$ has $0_{u}^{+}$ symmetry, confirming the original assignment of Zelevinsky et al. [3].

Coriolis mixing is a significant factor in the boundstate energies close to the ${ }^{1} S_{0}+{ }^{3} P_{1}$ limit in strontium. Not 
surprisingly, the energy shift by this mixing is dependent on the mixing angle $\theta$. This is especially important for the very long-range top bound states: for example, without Coriolis mixing, the theoretical energy for the $-0.435-\mathrm{MHz}$ level in ${ }^{88} \mathrm{Sr}$ is $-0.160 \mathrm{MHz}$. An even more extreme case is the top bound state in ${ }^{84} \mathrm{Sr}$ where the theoretical binding energy would be $-37 \mathrm{kHz}$ as opposed to the experimental value of $-0.32 \mathrm{MHz}$. Moreover, Coriolis mixing is especially strong when two energy levels of $0_{u}^{+}$and $1_{u}$ coincide, as is the case of the two energy levels in ${ }^{88} \mathrm{Sr}$ at -8200 and $-8430 \mathrm{MHz}$. The mixing angle $\theta=23^{\circ}$ for the $-8430-\mathrm{MHz}$ state, indicating particularly strong mixing, which again significantly influences the binding energy. In fact, without Coriolis mixing the respective theoretical energies are -8234 and $-8394 \mathrm{MHz}$, missing the experimental line positions by over $30 \mathrm{MHz}$. These two states are shown in the upper part of Fig. 2. The remaining bound states have mixing angles of about $5^{\circ}$ for $0_{u}^{+}$states and $\approx 95^{\circ}$ for $1_{u}$ symmetry and therefore are relatively pure, as shown for the $-3463-\mathrm{MHz}\left(0_{u}^{+}\right)$and $-2684-\mathrm{MHz}\left(1_{u}\right)$ states in the lower part of Fig. 2.

Our theoretical model provides an improved description of the nonadiabatic Coriolis mixing between the $0_{u}^{+}$and $1_{u}$ states. Recently, McGuyer et al. [4] gave experimentally determined linear Zeeman coefficients $g$ for the ${ }^{88} \mathrm{Sr}$ photoassociation lines, as well as their theoretical counterparts. The Zeeman coefficients

$$
g=g_{a t}\left(\frac{\sin ^{2} \theta}{J(J+1)}+\frac{\sin 2 \theta}{\sqrt{(J(J+1))}} P_{01}\right)
$$

are highly sensitive both to the mixing angle $\theta$ and the overlap $P_{01}$ of the components $\tilde{\Psi}\left(0_{u}^{+} ; R\right)$ and $\tilde{\Psi}\left(1_{u} ; R\right)$ :

$$
P_{01}=\int_{0}^{\infty} \tilde{\Psi}\left(0_{u}^{+} ; R\right) \tilde{\Psi}\left(1_{u} ; R\right) d R .
$$

The atomic $g$ factor $g_{a t}=1.5$ for the ${ }^{3} P_{1}$ electronic state.

A comparison of experimental and theoretical Zeeman $g$ factors from Ref. [4] with our values calculated with Eq. (10) is given in Table I. For relatively pure $0_{u}^{+}$and $1_{u}$ energy levels, our $g$ factor agrees very well with the theoretical values in [4]. In the case, however, of the top bound state at $-0.4 \mathrm{MHz}$ our theoretical value is slightly closer to the one obtained in experiment. The most striking improvement is seen in the case of the two strongly Coriolis-mixed bound states at -8200 and $-8430 \mathrm{MHz}$. As noted previously, these two energy levels are notoriously difficult to describe theoretically and the model in [4] fails to reproduce their experimental $g$ factors. Our model reduces the discrepancy between theory and experiment by a factor of 3 and while our model still does not fit to within experimental accuracy, it at least gives qualitative agreement. Since our mixing angles $\theta$ are in very good agreement, we attribute this improvement to the better description of the wave-function overlap $P_{01}$, which was a necessary condition to correctly reproduce the impact of Coriolis mixing on the positions of these two energy levels. This further corroborates the validity of our long-range van der Waals $C_{6}, C_{8}$, and resonant dipole $C_{3}$ coefficients. For completeness, we also provided our Zeeman $g$ factors for the remaining PA lines, but no experimental data are currently available to compare.

\section{MASS SCALING PERTURBED}

\section{A. Single-channel mass scaling}

A mass-scaled model is an interaction model that is capable of reproducing the energy levels for all isotopomers of a given molecule by only changing the reduced mass. By its nature, photoassociation spectroscopy is relatively insensitive to the details of the short-range atomic interaction as it is predominantly used to measure the energies of bound states very close to the dissociation limit. This can be understood using the following simple reasoning. The energy splittings between the vibrational states (on the order of $1 \mathrm{GHz}$ ) are very small compared to the depth of the potential curve (tens of $\mathrm{THz}$ ). Consequently, bound states close to the dissociation limit share the same short-range wave function (to within an amplitude constant) and only differ in the long range, where even such small energy differences will influence the location of the outer classical turning point and the longrange wave function. Therefore, to a certain approximation, the vibrational splittings would be determined by the longrange potential parameters, like $C_{6}$ and $C_{3}$ in our case. On the other hand, the starting point of the vibrational state ladder would be determined by the vibrational wave function's phase $\phi$, as required by the Bohr-Sommerfeld quantization condition. Such a philosophy is the basis for the famous analytic expression for the $s$-wave scattering length of an $R^{-n}$ potential [57], as well as the semiclassical LeRoy-Bernstein formulas [58].

As a consequence of the above reasoning, obtaining mass scaling in a single-channel model would only require a potential curve with the correct long-range part and the right behavior of the total phase $\phi$ as a function of the reduced mass. Such an approach has been discussed in detail in [59]. In the case of a single potential curve, the total phase can be well approximated using the WKB integral

$$
\phi=\frac{1}{\hbar} \int_{R_{\mathrm{in}}}^{\infty} \sqrt{-2 \mu V(R)} d R,
$$

where $R_{\text {in }}$ is the location of the inner classical turning point and $V$ is the interaction potential curve. It is evident that in this approach the total phase $\phi$ is proportional to $\sqrt{\mu}$ as long as the potential $V$ is mass independent. In this paper, we will assume that the potential $V$ is the same for all isotopes.

Following the Bohr-Sommerfeld quantization condition, the quantity $n=\phi / \pi$ is closely related to the number of bound states supported by the interaction potential. On the other hand, the positions of the vibrational states only depend on the fractional part $\Delta n=n-\lfloor n\rfloor$. Slowly increasing $n$, by e.g., adjusting the depth of the potential curve, causes the bound state energies to shift deeper into the potential well. At some point this will cause a new bound state at the dissociation limit to emerge. Increasing $n$ by 1 (or, equivalently, the total phase $\phi$ by $\pi$ ) would amount to adding exactly one vibrational state to the potential, with the weakly bound states having similar vibrational energies as previously. If we assume that the interaction potential $V$ is mass independent, then the quantity $n$ is proportional to $\sqrt{\mu}$. In this picture, mass scaling would amount to finding a potential curve which offers correct values of $\Delta n$ for each isotope. This can be achieved by simply fixing the correct number of bound states supported by the interaction 


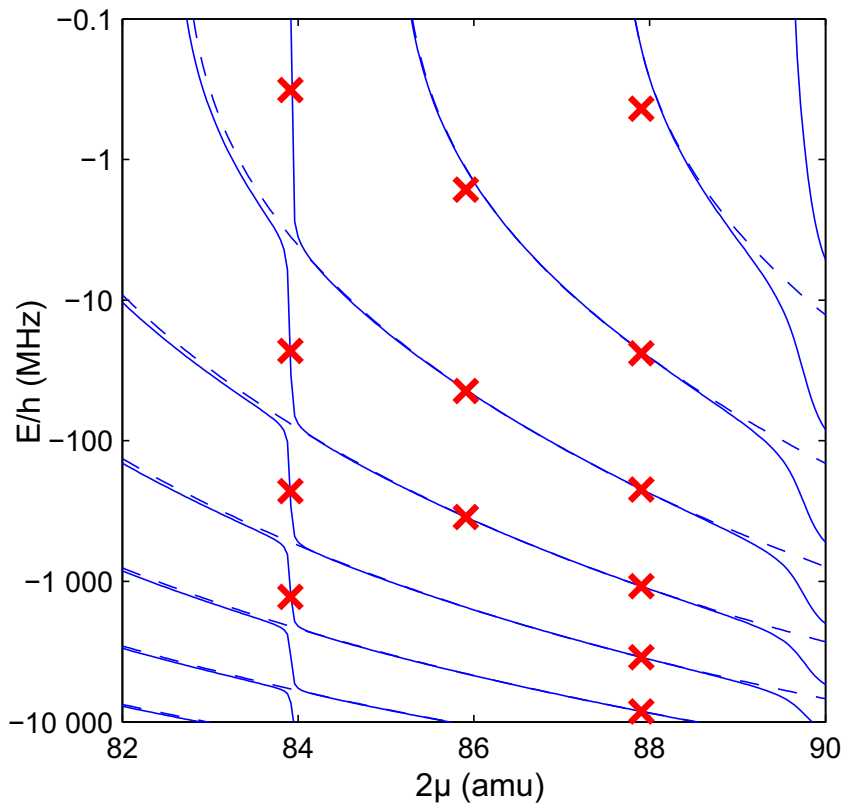

FIG. 3. (Color online) Theoretical $0_{u}^{+}$energy levels as a function of the reduced mass $\mu$. The blue dashed lines are calculated using the ${ }^{3} \Pi_{u}$ potential curve alone which is only capable of mass scaling between two isotopes at a time: ${ }^{86} \mathrm{Sr}$ and ${ }^{88} \mathrm{Sr}$ in this case. The solid lines show the results for a model that also includes the short-range crossing with a ${ }^{1} S_{0}+{ }^{1} D_{2}{ }^{1} \Sigma_{u}^{+}$potential, as shown in Fig. 4 . The latter model is capable of reproducing the experimental bound-state energies (marked as red crosses) for all isotopes. In this model, a short-range ${ }^{1} \Sigma_{u}^{+}$perturbing state crosses the ${ }^{1} S_{0}+{ }^{3} P_{1}$ dissociation limit at $2 \mu \approx 84$ amu causing a sudden departure from the usual mass scaling behavior.

potential. Such a strategy has been successfully implemented in a number of PA investigations $[7,59,60]$.

In our case the long-range interactions near the ${ }^{1} S_{0}+{ }^{3} P_{1}$ asymptote are described by two Hund's case (c) potentials $0_{u}^{+}$ and $1_{u}$ coupled by Coriolis mixing. We note, however, that this mixing is relatively weak and only provides a small correction to most bound-state energies. In fact, foregoing the Coriolis mixing altogether does not change the number of bound states supported by the two potentials together. Therefore, mass scaling of both series can to some extent be treated separately. We will first consider the mass scaling of energy levels of $\mathrm{O}_{u}^{+}$symmetry, for which most of the experimental data were collected. The final step will be the addition of the $1_{u}$ states to the model.

Figure 3 shows the $0_{u}^{+}$bound-state energies as a function of the reduced mass. By changing the $\alpha\left({ }^{3} \Pi_{u}\right)$ potential parameter, we have found an appropriate $0_{u}^{+}$potential depth (and consequently $\phi$ ) that correctly translated the wave-function phase between the isotopes ${ }^{86} \mathrm{Sr}$ and ${ }^{88} \mathrm{Sr}$. The results are shown in Fig. 3 as dashed lines. We have found that while our model fits the experimental bound-state energies for ${ }^{86} \mathrm{Sr}$ and ${ }^{88} \mathrm{Sr}$ to within $1 \mathrm{MHz}$ (with the exception of the strongly Coriolis-mixed $-8430-\mathrm{MHz}$ state), it is in shocking disagreement with the experimental data for ${ }^{84} \mathrm{Sr}$.

This clear failure of mass scaling can be easily explained a posteriori using the simple picture described earlier. The fractional part $\Delta n$ of the total WKB phase $\phi / \pi$ of the $0_{u}^{+}$state [computed by applying Eq. (12) to $\mathcal{V}\left({ }^{3} \Pi_{u} ; R\right)$ ] for the best-fit models from Sec. III is $\Delta n=0.3928,0.6003$, and 0.3245 for ${ }^{88} \mathrm{Sr},{ }^{86} \mathrm{Sr}$, and ${ }^{84} \mathrm{Sr}$, respectively. In the case of a single potential curve, $n$ scales linearly with $\sqrt{\mu}$. The model shown in Fig. 3 shows bound-state energies calculated using a potential that has $n=69.3928$ and mass scales, correctly, to $n=$ 68.6003 for ${ }^{86} \mathrm{Sr}$, which fits the observed line positions. Note that this model supports one bound state fewer for the latter isotope. For the ${ }^{84} \mathrm{Sr}$ isotope, however, mass scaling gives $n=$ 67.7988 , but this fails to predict the observed line positions.

Similar mass scaling can be obtained for other numbers of bound states, but in all cases only for two isotopes at a time. For example, in the physically reasonable range of 50 to 150 supported bound states, mass scaling between ${ }^{88} \mathrm{Sr}$ and ${ }^{86} \mathrm{Sr}$ is obtained only for 69 vibrational states, while for ${ }^{88} \mathrm{Sr}$ and ${ }^{84} \mathrm{Sr}$ the correct phases can be obtained for 89 or 133 states. Finally, for the ${ }^{86} \mathrm{Sr}-{ }^{84} \mathrm{Sr}$ pair a potential curve with 111 bound states would support mass scaling. Clearly, none of these numbers overlap. In fact, within this simple approximation no single potential curve supporting less than 300 vibrational states could support mass scaling between all three isotopes. For comparison, an unmodified ${ }^{3} \Pi_{u}$ curve from [44], as used in our model in Sec. III, supports a total of 72 bound states for $J=1$ in ${ }^{88} \mathrm{Sr}$.

\section{B. The avoided crossing}

It has recently been established, both experimentally [18] and theoretically [44], that the ${ }^{3} \Pi_{u}$ potential which supports the Hund's case (c) $0_{u}^{+}$potential correlating to the ${ }^{1} S_{0}+{ }^{3} P_{1}$ asymptote has a strong short-range avoided crossing with a ${ }^{1} \Sigma_{u}^{+}$curve which corresponds to the much higher ${ }^{1} S_{0}+{ }^{1} D_{2}$ asymptote. The resulting ${ }^{1} S_{0}+{ }^{3} P_{1} O_{u}^{+}$curve, as shown in Fig. 4, has several intriguing characteristics: its depth is defined almost solely by the depth of the ${ }^{1} \Sigma_{u}^{+}$potential and it enables nuclear motion at shorter distances than the ${ }^{3} \Pi_{u}$ curve alone. Skomorowski et al. [44] have analyzed the composition of bound states in the $0_{u}^{+}$state. The deepest bound states in the potential well are comprised primarily of the ${ }^{1} \Sigma_{u}^{+}$state, as expected given their energies are below the minimum of the ${ }^{3} \Pi_{u}$ curve. As we move up the bound-state ladder, the bound states become seemingly erratic mixtures of the ${ }^{1} \Sigma_{u}^{+}$and ${ }^{3} \Pi_{u}$ states. Finally, the bound states closest to the dissociation limit (and so far investigated by photoassociation spectroscopy) are nearly pure ${ }^{3} \Pi_{u}$ states, which explains why our long-range model alone was enough to quantitatively describe most of the observed vibrational spacings.

To test the influence of the ${ }^{1} \Sigma_{u}^{+}$state on the ${ }^{3} \Pi_{u}$ bound states and mass scaling, we use a two-channel model that includes both potentials:

$$
V_{\mathrm{int}}=\left(\begin{array}{cc}
\mathcal{V}\left({ }^{3} \Pi_{u} ; R\right)-C_{3,0} R^{-3} & \sigma \xi_{1}(R) \\
\sigma \xi_{1}(R) & \mathcal{V}\left({ }^{1} \Sigma_{u}^{+} ; R\right)+\Delta E
\end{array}\right),
$$

where, similar to Sec. III, $\mathcal{V}\left({ }^{1} \Sigma_{u}^{+} ; R\right)=V\left(\mathrm{~A}^{1} \Sigma_{u}^{+} ; R\right)-$ $V\left(\mathrm{~A}^{1} \Sigma_{u}^{+} ; \infty\right)$. This model obviously lacks the $1_{u}$ curve and, consequently, it does not include Coriolis mixing, limiting its accuracy to about $1 \mathrm{MHz}$ for bound states with small Coriolis-mixing angles. The diagonal rotational terms are $B(R)[J(J+1)+2]$ and $B(R) J(J+1)$, respectively. The 


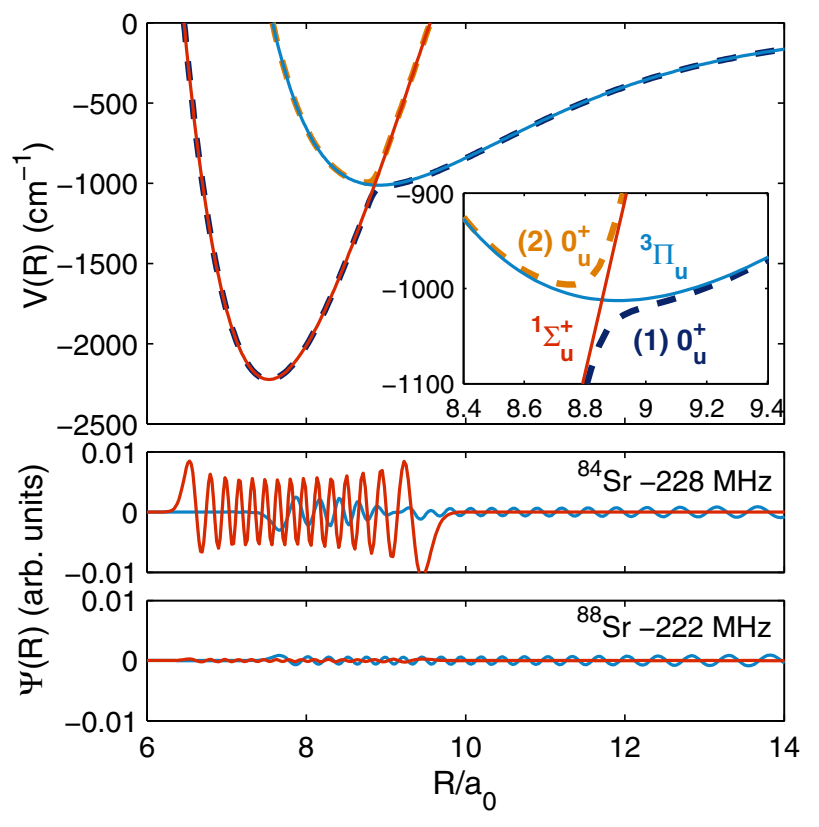

FIG. 4. (Color online) Avoided crossing between the ${ }^{1} S_{0}+$ ${ }^{3} P_{1}{ }^{3} \Pi_{u}$ and ${ }^{1} S_{0}+{ }^{1} D_{2}{ }^{1} \Sigma_{u}^{+}$potential curves. The strong spin-orbit coupling between these two potential curves causes anomalies in the mass-scaling behavior of the photoassociation spectra near strontium intercombination line. In fact, a model that only includes the potential curve directly supporting the $0_{u}^{+}$bound states close to the ${ }^{1} S_{0}+{ }^{3} P_{1}$ dissociation limit is incapable of properly describing mass scaling between the three bosonic isotopes in strontium.

potential $V\left({ }^{3} \Pi_{u} ; R\right)$ has the same parameters as the one from Sec. III except for $\alpha\left({ }^{3} \Pi_{u}\right)$, which we use to adjust the quantum defect. The $V\left(\mathrm{~A}^{1} \Sigma_{u}^{+} ; R\right)$ potential and spin-orbit coupling function $\xi_{1}(R)$ is the same as in [44]. Again, we model the influence of the ${ }^{1} S_{0}+{ }^{1} P_{1}{ }^{1} \Sigma_{u}^{+}$curve by manually adding the resonant dipole $-C_{3,0} R^{-3}$ term. The two additional fitted parameters are $\sigma \approx 0.42$, which enabled the scaling of the spin-orbit mixing between the two potential curves, and $\Delta E \approx 6250 \mathrm{~cm}^{-1}$ which controls the splitting between the ${ }^{3} P_{1}$ and ${ }^{1} D_{2}$ atomic states. We use the latter parameter to fix the position of the perturbing short-range ${ }^{1} \Sigma_{u}^{+}$bound state with respect to the ${ }^{1} S_{0}+{ }^{3} P_{1}$ asymptote while retaining the original shape of the ${ }^{1} \Sigma_{u}^{+}$potential. As was the case previously (Sec. III), the theoretical energy levels are obtained by solving the coupled-channel Schrödinger equation for the above potential matrix.
The solid lines in Fig. 3 show the theoretical bound-state energies calculated using the above two-channel model fitted to the experimental $0_{u}^{+}$data for all isotopes using only three parameters: $\alpha\left({ }^{3} \Pi_{u}\right)$, which as previously we used to adjust the short-range wave-function phase, the mixing parameter $\sigma$ that scales the ab initio [44] spin-orbit coupling function and the relative positions of the two potential curves $\Delta E$. The long-range parameters $C_{6}\left({ }^{3} \Pi_{u}\right)$ and $C_{3,0}$ are shared with the previously discussed long-range model from Sec. III. Apart from the ${ }^{84} \mathrm{Sr}-1288-\mathrm{MHz}$ state discussed in detail in Sec. IV, and the strongly Coriolis-mixed $-8430-\mathrm{MHz}$ state in ${ }^{88} \mathrm{Sr}$, the energy levels are reproduced to within $1 \mathrm{MHz}$. We note that the fitting itself was a very difficult process due to the seemingly erratic behavior of the model. This is caused by the mixing between the two diabatic potential curves ${ }^{3} \Pi_{u}$ and ${ }^{1} \Sigma_{u}^{+}$being strongly dependent on the relative phases of the two components $\Psi\left({ }^{3} \Pi_{u}\right)$ and $\Psi\left({ }^{1} \Sigma_{u}^{+}\right)$of the coupled-channel wave function. This is shown in the bottom part of Fig. 4. In our fit the $-222-\mathrm{MHz}$ state of ${ }^{88} \mathrm{Sr}$ is a nearly pure ${ }^{3} \Pi_{u}$ state, while the $-228-\mathrm{MHz}$ level in ${ }^{84} \mathrm{Sr}$ is strongly influenced by a short-range ${ }^{1} \Sigma_{u}^{+}$component. Such a dramatic change of ${ }^{1} \Sigma_{u}^{+}$component amplitude is likely the result of a bound state of this symmetry coinciding with the ${ }^{1} S_{0}+{ }^{3} P_{1}$ asymptote in ${ }^{84} \mathrm{Sr}$.

The mass-scaling behavior of this model (solid lines in Fig. 3) is similar to the single-channel case (dashed lines) for the range of reduced masses of about $2 \mu \approx 85 \ldots 89 \mathrm{u}$. However, when a short-range ${ }^{1} \Sigma_{u}^{+}$bound state crosses the ${ }^{1} S_{0}+{ }^{3} P_{1}$ dissociation limit, there is a sudden resonant departure as seen near $2 \mu \approx 84 \mathrm{u}$ and $2 \mu \approx 90 \mathrm{u}$. Since the spin-orbit mixing is relatively strong, the width of this resonant structure is large and encompasses all bound states close to the dissociation limit together, resulting in an apparent change in the quantum defect. This explains why the vibrational spacings alone can be described by a single-channel model even when the mixing is large. A comparison of the experimental $0_{u}^{+}$energy levels with ones calculated from our mass-scaled model is shown in Table IV. The accuracy of the model is about $1 \mathrm{MHz}$ for the majority of the experimental data and is mostly limited by the lack of Coriolis mixing.

\section{Impact on vibrational spacings}

The available photoassociation data for $J=1$ states in ${ }^{88} \mathrm{Sr}$ and ${ }^{86} \mathrm{Sr}$ can be well described by a simple model that only includes the ${ }^{3} \Pi_{u}\left(0_{u}^{+}\right)$curve. Consequently, significant mixing

TABLE IV. A comparison of experimental $0_{u}^{+}$bound-state energies with predictions of our mass-scaled model that includes the strong spin-orbit coupling between the ${ }^{1} S_{0}+{ }^{3} P_{1}{ }^{3} \Pi_{u}$ curve which directly supports the $0_{u}^{+}$series observed in PA experiments and the ${ }^{1} S_{0}+{ }^{1} D_{2}{ }^{1} \Sigma_{u}^{+}$ curve. See text.

\begin{tabular}{|c|c|c|c|c|c|c|}
\hline \multirow[b]{2}{*}{$v$} & \multicolumn{2}{|c|}{${ }^{88} \mathrm{Sr}$} & \multicolumn{2}{|c|}{${ }^{86} \mathrm{Sr}$} & \multicolumn{2}{|c|}{${ }^{84} \mathrm{Sr}$} \\
\hline & Expt. & Theory & Expt. & Theory & Expt. & Theory \\
\hline-1 & -0.435 & -0.164 & -1.633 & -1.169 & -0.320 & -0.071 \\
\hline-2 & -23.932 & -23.133 & -44.246 & -43.467 & -23.010 & -23.208 \\
\hline-3 & -222.161 & -221.137 & -348.742 & -349.115 & -228.380 & -228.173 \\
\hline-4 & -1084.093 & -1083.353 & & & -1288.290 & -1062.636 \\
\hline-5 & -3463.280 & -3463.857 & & & & \\
\hline-6 & -8429.650 & -8410.027 & & & & \\
\hline
\end{tabular}


TABLE V. A comparison of theoretical energy levels of $0_{u}^{+}$ symmetry calculated with ("Perturbed") and without ("Adiabatic") the spin-orbit mixing between the ${ }^{1} \Sigma_{u}^{+}$and ${ }^{3} \Pi_{u}$ curves. The quantum defect has been adjusted in the adiabatic case so that the energy of the $v=-3$ bound state strictly matches the one predicted by the two-channel model. This ensures that any differences between the energy level predictions are caused by the way the models are constructed rather than quantum defects. In our model, the ${ }^{84} \mathrm{Sr}$ bound states close to the dissociation limit are significantly perturbed by this mixing, which changes their vibrational splittings significantly. On the other hand, the ${ }^{88} \mathrm{Sr}$ bound states remain relatively unchanged. This behavior can be used in the future to ascertain the location of the perturbing states. All values are given in MHz.

\begin{tabular}{lrrrrr}
\hline \hline & \multicolumn{2}{c}{${ }^{84} \mathrm{Sr}$} & & \multicolumn{2}{c}{${ }^{88} \mathrm{Sr}$} \\
\cline { 2 - 3 } \cline { 5 - 6 }$v$ & Perturbed & Adiabatic & & Perturbed & Adiabatic \\
\hline-3 & -228.4 & -228.4 & & -222.2 & -222.2 \\
-4 & -1063.3 & -1147.6 & & -1087.0 & -1086.8 \\
-5 & -3087.8 & -3704.1 & & -3472.5 & -3471.2 \\
-6 & -6983.4 & -9024.2 & & -8426.4 & -8419.9 \\
-7 & -13987.8 & -18316.1 & & -17080.6 & -17056.0 \\
\hline \hline
\end{tabular}

between ${ }^{3} \Pi_{u}$ and ${ }^{1} \Sigma_{u}^{+}$must occur for bound states deeper in the well. Perturbation theory dictates that the size of the ${ }^{1} \Sigma_{u}^{+}$ contribution to an otherwise ${ }^{3} \Pi_{u}$ bound state increases in the vicinity of a ${ }^{1} \Sigma_{u}^{+}$bound state.

A comparison of the bound-state energies predicted by our adiabatic (single-channel) and perturbed (two-channel massscaled) model is shown in Table V for isotopes ${ }^{84} \mathrm{Sr}$ and ${ }^{88} \mathrm{Sr}$. For both isotopes we have adjusted the wave-function phases so that the energies of the $v=-3$ bound-state energies match. This way, we ensure a fair comparison of vibrational splittings. In our model, the perturbing short-range ${ }^{1} \Sigma_{u}^{+}$bound state crosses the ${ }^{1} S_{0}+{ }^{3} P_{1}$ dissociation limit when the reduced mass $2 \mu \approx 84 \mathrm{u}$. Consequently, there are significant differences in the bound-state energies for $v=-5$ and deeper states. On the other hand, in the case of ${ }^{88} \mathrm{Sr}$, the differences between the models are about an order of magnitude smaller. This shows that it will be possible to experimentally determine which of the isotopes has its ${ }^{1} S_{0}+{ }^{3} P_{1}$ asymptote perturbed. In our model ${ }^{84} \mathrm{Sr}$ is the perturbed isotope, but given our limited knowledge of the strontium PA spectra, ${ }^{86} \mathrm{Sr}$ could be the perturbed isotope as well. The subtle discrepancies between the ${ }^{86} \mathrm{Sr}$ experimental data and our theoretical model from Sec. III might hint toward this hypothesis. This uncertainty can be resolved experimentally by simply measuring the bound-state energies deeper in the potential well. One of the isotopes should clearly have its vibrational spacings incompatible with an interaction model that only includes the channels belonging to the ${ }^{1} S_{0}+{ }^{3} P_{1}$ asymptote.

\section{Mass scaling of $\mathbf{1}_{u}$ energy levels and the three-channel model}

The last step in the construction of our mass-scaled model is the introduction of the $1_{u}$ molecular state. The interaction potential matrix, as expressed in the

$$
\begin{aligned}
& \left\{0_{u}^{+}\left({ }^{3} \Pi_{u}\right), 0_{u}^{+}\left({ }^{1} \Sigma_{u}^{+}\right), 1_{u}\left({ }^{3} \Sigma_{u}^{+},{ }^{3} \Pi_{u}\right)\right\} \text { Hund's case }(\mathrm{c}) \text { basis, is now } \\
& V_{\text {int }}=\left(\begin{array}{ccc}
\mathcal{V}\left({ }^{3} \Pi_{u} ; R\right)- & \sigma \xi_{1}(R) & 0 \\
-C_{3,0} R^{-3} & \mathcal{V}\left({ }^{1} \Sigma_{u}^{+} ; R\right)+ & \\
\sigma \xi_{1}(R) & +\Delta E & 0 \\
& 0 & \begin{array}{c}
\frac{1}{2}\left(\mathcal{V}\left({ }^{3} \Pi_{u} ; R\right)+\right. \\
\left.+\mathcal{V}\left({ }^{3} \Sigma_{u}^{+} ; R\right)\right)- \\
0
\end{array} \\
& & -C_{3,1} / R^{3}
\end{array}\right)
\end{aligned}
$$

Similarly, the rotational Hamiltonian

$$
V_{\mathrm{rot}}=\left(\begin{array}{ccc}
J(J+1)+2 & 0 & -\sqrt{4 J(J+1)} \\
0 & J(J+1) & 0 \\
-\sqrt{4 J(J+1)} & 0 & J(J+1)
\end{array}\right)
$$

now contains the Coriolis-mixing terms like the long-range interaction model from Sec. III. The kinetic Hamiltonian $T$ is again diagonal and the energy levels are calculated by solving the set of coupled Schrödinger equations $H \Psi=E \Psi$ for $H=$ $T+V_{\text {int }}+V_{\text {rot }}$ and the three-channel wave function $\Psi$.

At this point, reaching a mass-scaled model for both $0_{u}^{+}$ and $1_{u}$ energy levels is relatively straightforward. Since the Coriolis mixing is a small correction to the $0_{u}^{+}$energies, we can hope that the mass-scaling model utilizing the ${ }^{3} \Pi_{u^{-}}{ }^{1} \Sigma_{u}^{+}$ curve crossing will stand except for cosmetic corrections to its parameters.

Experimental $1_{u}$ energy level data are, to date, only available for the ${ }^{88} \mathrm{Sr}$ and ${ }^{86} \mathrm{Sr}$ isotopes. We can therefore apply the single-channel mass-scaling strategy as described in Sec. VI A, that is, fix the right number of bound states supported by the $1_{u}$ state. To this end, we have modified the short-range $\alpha\left({ }^{3} \Sigma_{u}^{+}\right)$parameter, which only affects the $1_{u}$ quantum defect. Finally, we have run a least-squares optimization for both quantum defect $\alpha$ parameters and the curve crossing parameters $\Delta E$ and $\sigma$ in order to obtain the final fit. All of the long-range parameters $\left(C_{3,0}\right.$, as well as $C_{6}$ and $C_{8}$ for both potential curves) remain the same.

A comparison of experimental energy levels for all strontium isotopes with those predicted by our final three-channel model is shown in Table VI. Apart from the ${ }^{84} \mathrm{Sr}-1288-\mathrm{MHz}$ state discussed at length in Sec. IV and not included in this table, the theoretical energy levels match their experimental counterparts to about $0.5 \mathrm{MHz}$ on average. Mass scaling between states of $1_{u}$ symmetry is acceptable, although we note that theoretical position of the ${ }^{86} \mathrm{Sr}$ state at $-159 \mathrm{MHz}$ is shifted by about $3 \mathrm{MHz}$. We have attempted to improve the accuracy of this fit by changing the number of bound states supported by the $1_{u}$ curve, but this resulted in a similar shift in the other direction.

For each bound state, we also list channel contributions $c_{i}$ calculated using $c_{i}=\int_{0}^{\infty}\left|\Psi_{i}(R)\right|^{2} d R$ and normalized via $c_{1}+c_{2}+c_{3}=1$. The channel contributions again show the nonadiabatic effects described throughout this paper. For instance, the Coriolis mixing between the ${ }^{1} S_{0}+{ }^{3} P_{1} O_{u}^{+}$and $1_{u}$ states is clearly visible in the top $0_{u}^{+}$bound states of ${ }^{84} \mathrm{Sr}$ and ${ }^{88} \mathrm{Sr}$, where the $1_{u}$ contribution reaches about $10 \%$. 
TABLE VI. Final $0_{u}^{+}$and $1_{u}$ bound-state energies as calculated from our three-channel mass-scaled model described in Sec. VI D. Channel contributions are also given.

\begin{tabular}{|c|c|c|c|c|c|}
\hline \multirow[b]{2}{*}{ Isotope } & \multicolumn{2}{|c|}{ Energy levels (MHz) } & \multicolumn{3}{|c|}{ Channel contributions $(\%)$} \\
\hline & Experiment & Theory & $0_{u}^{+}\left({ }^{3} \Pi_{u}\right)$ & $0_{u}^{+}\left({ }^{1} \Sigma_{u}^{+}\right)$ & $\overline{1_{u}\left({ }^{3} \Sigma_{u}^{+},{ }^{3} \Pi_{u}\right)}$ \\
\hline${ }^{88} \mathrm{Sr}$ & $\begin{array}{r}-0.435 \\
-23.932 \\
-222.161 \\
-353.236 \\
-1084.093 \\
-2683.722 \\
-3463.280 \\
-8200.163 \\
-8429.650\end{array}$ & $\begin{array}{r}-0.426 \\
-23.856 \\
-222.021 \\
-352.934 \\
-1083.648 \\
-2683.117 \\
-3463.434 \\
-8199.777 \\
-8431.909\end{array}$ & $\begin{array}{r}90.991 \\
98.881 \\
99.499 \\
0.462 \\
99.597 \\
0.730 \\
99.252 \\
14.194 \\
85.764\end{array}$ & $\begin{array}{l}0.000 \\
0.000 \\
0.003 \\
0.000 \\
0.009 \\
0.000 \\
0.022 \\
0.006 \\
0.036\end{array}$ & $\begin{array}{r}9.009 \\
1.118 \\
0.498 \\
99.538 \\
0.393 \\
99.270 \\
0.726 \\
85.800 \\
14.200\end{array}$ \\
\hline${ }^{86} \mathrm{Sr}$ & $\begin{array}{r}-1.633 \\
-44.246 \\
-159.984 \\
-348.742\end{array}$ & $\begin{array}{r}-1.535 \\
-43.864 \\
-163.296 \\
-348.925\end{array}$ & $\begin{array}{r}94.991 \\
98.785 \\
1.628 \\
98.624\end{array}$ & $\begin{array}{l}0.000 \\
0.000 \\
0.000 \\
0.001\end{array}$ & $\begin{array}{r}5.009 \\
1.215 \\
98.372 \\
1.375\end{array}$ \\
\hline${ }^{84} \mathrm{Sr}$ & $\begin{array}{r}-0.320 \\
-23.010 \\
-228.380\end{array}$ & $\begin{array}{r}-0.341 \\
-23.541 \\
-32.855 \\
-228.259 \\
-1023.648 \\
-1472.635\end{array}$ & $\begin{array}{r}89.470 \\
89.183 \\
10.125 \\
95.130 \\
88.505 \\
0.206\end{array}$ & $\begin{array}{r}0.025 \\
0.548 \\
0.074 \\
3.912 \\
11.229 \\
0.135\end{array}$ & $\begin{array}{r}10.505 \\
10.269 \\
89.800 \\
0.957 \\
0.266 \\
99.660\end{array}$ \\
\hline
\end{tabular}

Similarly the pair of $-8-\mathrm{GHz}$ states in ${ }^{88} \mathrm{Sr}$ is again very strongly mixed with mutual contributions as high as $14 \%$. On the other hand, the short-range mixing between the two $0_{u}^{+}$states only significantly affects the ${ }^{84} \mathrm{Sr}$ isotope with contributions up to $3.9 \%$ for the $-228-\mathrm{MHz}$ state while for the remaining isotopes it is below $0.04 \%$ and as such is insignificant.

\section{CONCLUSION}

In conclusion, we have measured the energies of four vibrational bound states of the ${ }^{86} \mathrm{Sr}$ molecule in an excited triplet state using photoassociation spectroscopy of ultracold strontium atoms. The obtained data complement previously reported photoassociation data for the remaining two bosonic isotopes of strontium: ${ }^{84} \mathrm{Sr}$ and ${ }^{88} \mathrm{Sr}$. We have provided an $a b$ initio based theoretical model that correctly describes Coriolis mixing between $0_{u}^{+}$and $1_{u}$ states. Only one of the previously reported energy levels, in ${ }^{84} \mathrm{Sr}$, is in qualitative disagreement with our theory and we have suggested that it may either be supported by a different potential curve, or be strongly perturbed. We have used our theoretical model to provide Zeeman $g$ factors for all of the considered photoassociation lines.

We have also shown, however, that a theoretical model that only takes into account the two potential curves correlating to the ${ }^{1} S_{0}+{ }^{3} P_{1}$ asymptote will fail to correctly describe the positions of energy levels for all isotopes via simple mass scaling, even despite being correct for one isotope at a time. We attribute that to the short-range avoided crossing between the ${ }^{1} S_{0}+{ }^{3} P_{1}{ }^{3} \Pi_{u}$ curve and a higher excited state ${ }^{1} S_{0}+{ }^{1} D_{2}{ }^{1} \Sigma_{u}^{+}$potential: a short-range ${ }^{1} \Sigma_{u}^{+}$bound state would perturb the positions of the series of states observed in photoassociation spectroscopy near the intercombination line. We have produced a three-channel model that take this mixing into account and is capable of reproducing the available $0_{u}^{+}$ and $1_{u}$ energy levels. Finally, we suggest that this theory can be tested by measuring more deeply bound vibrational states. For at least one isotope, the vibrational spacings should significantly depart from those predicted by the long-range model alone.

The results shown here will be important in the research concerning optical Feshbach resonances near the intercombination line in $\mathrm{Sr}$. The four resonances reported in the paper can now be used to control the interactions between ${ }^{86} \mathrm{Sr}$ atoms in a similar manner as it was done in ${ }^{88} \mathrm{Sr}[28,29]$. The mixing between states could also influence the possible use of $\mathrm{Sr}$ as a means for measuring changes in the $m_{p} / m_{e}$ ratio $[15,61]$. The findings here also provide a template for the description of mass scaling perturbed by short-range avoided crossings in other similar systems: a similar crossing between ${ }^{3} \Pi_{u}$ and ${ }^{1} \Sigma_{u}^{+}$potentials has already been found in the calcium system [62].

\section{ACKNOWLEDGMENTS}

The authors wish to thank P. Żuchowski and W. Skomorowski for their help with setting up the calculations and useful discussions. We are also grateful to S. G. Porsev, M. S. Safronova, and C. W. Clark for providing us with the results of their work before publication. M.Y., B.J.D., and T.C.K. acknowledge support from the Welch Foundation (Grants No. C-1579 and No. C-1669) and the National Science Foundation(Grants No. PHY-1205946 and No. PHY-1205973). This work has been partially supported by the FNP TEAM Programme Project Precise Optical Control and Metrology of Quantum Systems No. TEAM/2010-6/3 cofinanced by the European Union Regional Development Fund and is part of the ongoing research program of National Laboratory FAMO in Toruń, Poland.

\section{APPENDIX: FITTING PHOTOASSOCIATION SPECTRA}

Experiments determine the number of atoms in the trap after exposure to the PA laser at frequency $f$ for interaction time $t$. Assuming constant sample temperature, and loss described by $\dot{n}=-2 K n^{2}$, the time evolution of the number of atoms is given by

$$
N(t)=\frac{N_{0}}{1+2 N_{0} K t V_{2} / V_{1}^{2}},
$$

where $N_{0}$ is the number of atoms without applying PA beams, $K$ is the effective collision event rate constant, and $V_{q}(q=1$ and 2) are the effective volumes defined by

$$
V_{q}=\int d^{3} r e^{-q U(r) / k_{B} T},
$$

with the trap potential $U(r)$, the Boltzmann constant $k_{B}$, and the sample temperature $T$. For a high ratio of trap 


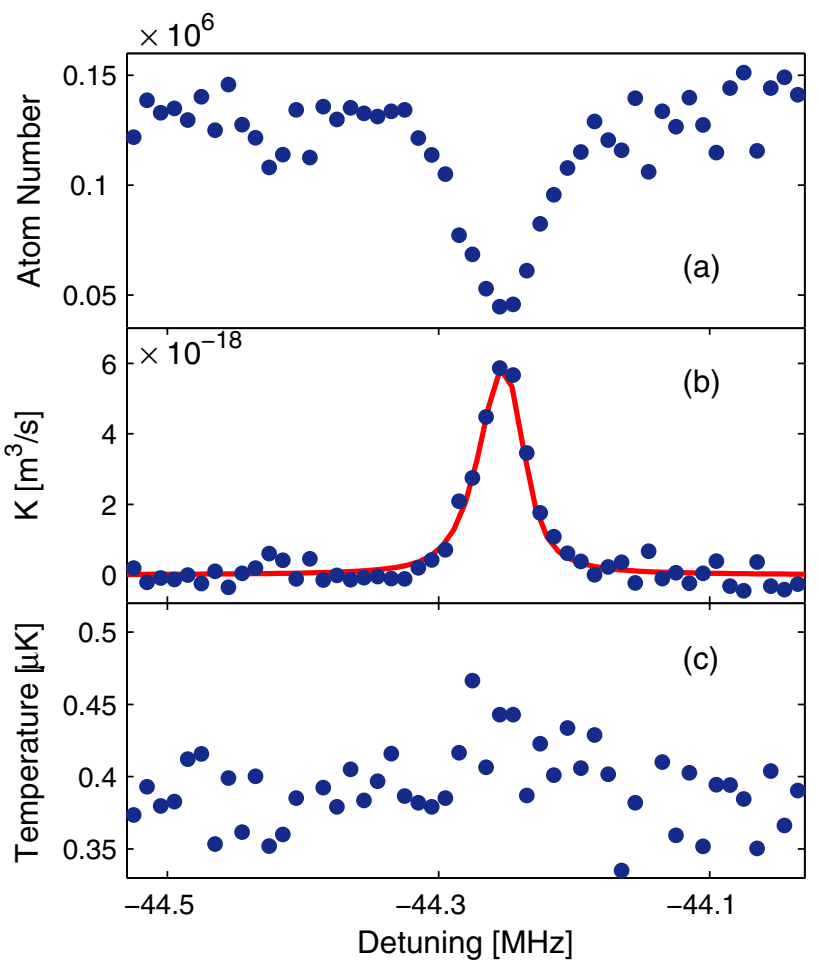

FIG. 5. (Color online) Spectroscopy of the $0_{u}^{+}(v=-2)$ PA line of ${ }^{86} \mathrm{Sr}$. (a) Atom number versus laser detuning from the one-photon ${ }^{1} S_{0}-{ }^{3} P_{1}$ atomic transition for interaction time $t=59.4 \mathrm{~ms}$. (b) The effective collision event rate constant derived from the atom loss using Eq. (A4). (c) Temperature versus detuning shows little variation, supporting the assumption of constant sample temperature.

depth to temperature $\varepsilon_{t} / k_{B} T$, the effective volumes can be approximated by

$$
V_{q}=\left(\frac{2 \pi k_{B} T}{q m \bar{\omega}^{2}}\right)^{3 / 2}
$$

where $m$ is the atomic mass, and $\bar{\omega}$ is the geometric mean of the angular oscillator frequencies of the trap. Equations (A1) and (A3) yield

$$
K=\frac{4}{t}\left(\frac{1}{N(t)}-\frac{1}{N_{0}}\right)\left(\frac{\pi k_{B} T}{m \bar{\omega}^{2}}\right)^{3 / 2}
$$

which allows direct determination of spectra of $K$ from the atom-loss spectra. Figure 5 is an example near the $0_{u}^{+}(v=$ -2) PA line of ${ }^{86} \mathrm{Sr}$.

The spectra of collision event rate constant $K$ [Fig. 5(b)] are fit using the formalism of Ciuryło et al. [10]. This accounts for Doppler broadening and photon recoil, which is necessary when the atomic temperature $T$ is lower than the atomic recoil temperature $T_{R}=(h / \lambda)^{2} /\left(m k_{B}\right)=460 \mathrm{nK}$, for wavelength of the PA laser $\lambda=689 \mathrm{~nm}$. In our experiments, the sample temperature is $T \sim 400 \mathrm{nK}$. In this regime, the collision event rate constant is given by [10]

$$
K=\frac{k_{B} T}{h Q_{T}} \int_{-\infty}^{+\infty} d y e^{-y^{2}} \int_{0}^{\infty} d x x e^{-x^{2}} \mathcal{L}\left(f, y, x^{2}\right)
$$

TABLE VII. Values of parameters of ${ }^{86} \mathrm{Sr}$ PA lines extracted from the experiment. Bound-state energies are given for the $v=-1,-$ $2,-3$ levels of $0_{u}^{+}$symmetry and the $v=-1$ level of $1_{u}$ symmetry, where $v$ counts levels down from the dissociation limit. These levels all have angular momentum $J=1$. We also report the optical lengths $l_{\text {opt }}$ for each of the measured $0_{u}^{+}$PA lines. The term $\eta^{\text {upper }}$ describes the additional molecular loss factor $[25,28]$ and $\gamma_{\text {laser }}^{\text {uper }}$ is the upper limit on the PA laser linewidth.

\begin{tabular}{lcrrrr}
\hline \hline$v$ & $\begin{array}{c}{ }^{86} \mathrm{Sr} \\
\text { Series }\end{array}$ & $v_{0}(\mathrm{MHz})$ & $l_{\text {opt }} / I\left[a_{\mathrm{B}} /\left(\mathrm{W} / \mathrm{cm}^{2}\right)\right]$ & $\eta^{\text {upper }}$ & $\begin{array}{c}\gamma_{\text {lasper }}^{\text {upper }} / 2 \pi \\
(\mathrm{kHz})\end{array}$ \\
\hline-1 & $0_{u}^{+}$ & $-1.633(10)$ & $(3.8 \sim 6.5) \times 10^{4}$ & 1.7 & 10.5 \\
-2 & $0_{u}^{+}$ & $-44.246(10)$ & $(1.2 \sim 1.8) \times 10^{4}$ & 1.5 & 7.5 \\
-1 & $1_{u}$ & $-159.984(50)$ & & & \\
-3 & $0_{u}^{+}$ & $-348.742(10)$ & $(5.7 \sim 10.3) \times 10^{2}$ & 1.8 & 12.0 \\
\hline \hline
\end{tabular}

where $x$ and $y$ are dimensionless variables, and

$$
\begin{aligned}
& \mathcal{L}\left(f, y, x^{2}\right) \\
& =\frac{\eta \gamma_{\mathrm{mol}} \gamma_{s} /(2 \pi)^{2}}{\left(f+y \Delta_{D}+x^{2} \Delta_{T}-E_{b} / h-E_{\mathrm{rec}} / h\right)^{2}+\left(\eta^{\prime} \gamma_{\mathrm{mol}} / 4 \pi\right)^{2}}
\end{aligned}
$$

with the thermal width $\Delta_{T}=k_{B} T / h$, the Doppler width $\Delta_{D}=\sqrt{k_{B} T / m} / \lambda$, the natural linewidth of the excited molecular level $\gamma_{\mathrm{mol}}=2 \pi \times 15 \mathrm{kHz}$, and the stimulated width $\gamma_{s}=2\left(\sqrt{2 \mu x^{2} \Delta_{T}} / \hbar\right) \gamma_{\mathrm{mol}} \ell_{\mathrm{opt}} \ll \gamma_{\mathrm{mol}}$, where $\ell_{\mathrm{opt}}$ is the optical length. Here, $Q_{T}=\left(2 \pi k_{B} T \mu / h^{2}\right)^{3 / 2}$ is the partition function for reduced mass $\mu=m / 2, h$ is Planck's constant, $E_{b}$ is the PA line center including the molecular ac Stark shift due to PA beams, and $E_{\text {rec }}=(h / \lambda)^{2} /(4 m)$ is the photon recoil energy of an isolated atom. The parameter $\eta \geqslant 1$ accounts for the extra molecular losses observed in OFR experiments [25,28], and $\eta^{\prime} \gamma_{\mathrm{mol}}=\eta \gamma_{\mathrm{mol}}+\gamma_{\text {laser }}$ with the linewidth of the PA laser $\gamma_{\text {laser }}$. We can only determine $E_{\mathrm{b}}, \eta^{\prime}=\eta+\gamma_{\text {laser }} / \gamma_{\mathrm{mol}}$, and $\eta \ell_{\mathrm{opt}}$, and cannot independently determine $\eta, \ell_{\mathrm{opt}}$, and $\gamma_{\text {laser }}$. Truncation of the integral over collision kinetic energy has been neglected.

An example of the fitting result is shown in Fig. 5(b). The line center shifts linearly with the PA laser intensity, and we obtain spectra under a range of conditions and extrapolate to zero intensity to obtain the unshifted resonance position $v_{0}$ (Table VII). Fitting determines $v_{0}$ with a precision of $5 \mathrm{kHz}$. There is additional systematic error in determining the laser detuning with respect to atomic resonance of $5 \mathrm{kHz}$, and we quote a total uncertainty of $10 \mathrm{kHz}$ (Table VII). The $v=-1,1_{u}$ binding energy is quoted with increased uncertainty because the data showed significant temperature variation, complicating the analysis. Further details of the experiment and the fitting procedure can be found in [63].

By linearly fitting the values of $\ell_{\mathrm{opt}}$ at different $I$, we extract $\ell_{\text {opt }} / I$. The upper limit of $\eta\left(\eta^{\text {upper }}\right)$ and the lower limit of $\ell_{\text {opt }}$ can be determined by fixing $\gamma_{\text {laser }}=0$, while the upper limit of $\gamma_{\text {laser }}\left(\gamma_{\text {laser }}^{\text {upper }}\right)$ and the upper limit of $\ell_{\text {opt }}$ can be determined by fixing $\eta=1$. Values of these parameters from the fitting are summarized in Table VII. 
[1] K. M. Jones, E. Tiesinga, P. D. Lett, and P. S. Julienne, Rev. Mod. Phys. 78, 483 (2006).

[2] M. Kahmann, E. Tiemann, O. Appel, U. Sterr, and F. Riehle, Phys. Rev. A 89, 023413 (2014).

[3] T. Zelevinsky, M. M. Boyd, A. D. Ludlow, T. Ido, J. Ye, R. Ciuryło, P. Naidon, and P. S. Julienne, Phys. Rev. Lett. 96, 203201 (2006).

[4] B. H. McGuyer, C. B. Osborn, M. McDonald, G. Reinaudi, W. Skomorowski, R. Moszynski, and T. Zelevinsky, Phys. Rev. Lett. 111, 243003 (2013).

[5] S. Stellmer, B. Pasquiou, R. Grimm, and F. Schreck, Phys. Rev. Lett. 109, 115302 (2012).

[6] S. Tojo, M. Kitagawa, K. Enomoto, Y. Kato, Y. Takasu, M. Kumakura, and Y. Takahashi, Phys. Rev. Lett. 96, 153201 (2006).

[7] M. Borkowski, R. Ciuryło, P. S. Julienne, S. Tojo, K. Enomoto, and Y. Takahashi, Phys. Rev. A 80, 012715 (2009).

[8] M. Borkowski, R. Ciurylo, P. S. Julienne, R. Yamazaki, H. Hara, K. Enomoto, S. Taie, S. Sugawa, Y. Takasu, and Y. Takahashi, Phys. Rev. A 84, 030702 (2011).

[9] Y. Takasu, Y. Saito, Y. Takahashi, M. Borkowski, R. Ciuryło, and P. S. Julienne, Phys. Rev. Lett. 108, 173002 (2012).

[10] R. Ciuryło, E. Tiesinga, S. Kotochigova, and P. S. Julienne, Phys. Rev. A 70, 062710 (2004).

[11] W. Skomorowski, R. Moszynski, and C. P. Koch, Phys. Rev. A 85, 043414 (2012).

[12] R. Ciuryło, E. Tiesinga, and P. S. Julienne, Phys. Rev. A 71, 030701 (2005)

[13] C. P. Koch and M. Shapiro, Chem. Rev. 112, 4928 (2012).

[14] M. Yan, B. J. DeSalvo, Y. Huang, P. Naidon, and T. C. Killian, Phys. Rev. Lett. 111, 150402 (2013).

[15] S. Kotochigova, T. Zelevinsky, and J. Ye, Phys. Rev. A 79, 012504 (2009).

[16] Y. N. Martinez de Escobar, P. G. Mickelson, P. Pellegrini, S. B. Nagel, A. Traverso, M. Yan, R. Côté, and T. C. Killian, Phys. Rev. A 78, 062708 (2008).

[17] A. Stein, H. Knöckel, and E. Tiemann, Phys. Rev. A 78, 042508 (2008).

[18] A. Stein, H. Knöckel, and E. Tiemann, Eur. Phys. J. D 57, 171 (2010).

[19] A. Stein, H. Knoeckel, and E. Tiemann, Eur. Phys. J. D 64, 227 (2011).

[20] P. O. Fedichev, Y. Kagan, G. V. Shlyapnikov, and J. T. M. Walraven, Phys. Rev. Lett. 77, 2913 (1996).

[21] J. L. Bohn and P. S. Julienne, Phys. Rev. A 56, 1486 (1997).

[22] C. Chin, R. Grimm, P. Julienne, and E. Tiesinga, Rev. Mod. Phys. 82, 1225 (2010).

[23] P. D. Lett, K. Helmerson, W. D. Phillips, L. P. Ratliff, S. L. Rolston, and M. E. Wagshul, Phys. Rev. Lett. 71, 2200 (1993).

[24] F. K. Fatemi, K. M. Jones, and P. D. Lett, Phys. Rev. Lett. 85, 4462 (2000).

[25] M. Theis, G. Thalhammer, K. Winkler, M. Hellwig, G. Ruff, R. Grimm, and J. H. Denschlag, Phys. Rev. Lett. 93, 123001 (2004).

[26] G. Thalhammer, M. Theis, K. Winkler, R. Grimm, and J. H. Denschlag, Phys. Rev. A 71, 033403 (2005).

[27] K. Enomoto, K. Kasa, M. Kitagawa, and Y. Takahashi, Phys. Rev. Lett. 101, 203201 (2008).
[28] S. Blatt, T. L. Nicholson, B. J. Bloom, J. R. Williams, J. W. Thomsen, P. S. Julienne, and J. Ye, Phys. Rev. Lett. 107, 073202 (2011).

[29] M. Yan, B. J. DeSalvo, B. Ramachandhran, H. Pu, and T. C. Killian, Phys. Rev. Lett. 110, 123201 (2013).

[30] P. G. Mickelson, Y. N. Martinez, A. D. Saenz, S. B. Nagel, Y. C. Chen, T. C. Killian, P. Pellegrini, and R. Côté, Phys. Rev. Lett. 95, 223002 (2005).

[31] T. Ido, Y. Isoya, and H. Katori, Phys. Rev. A 61, 061403 (2000).

[32] G. Ferrari, R. E. Drullinger, N. Poli, F. Sorrentino, and G. M. Tino, Phys. Rev. A 73, 023408 (2006).

[33] S. Stellmer, M. K. Tey, B. Huang, R. Grimm, and F. Schreck, Phys. Rev. Lett. 103, 200401 (2009).

[34] Y. N. Martinez de Escobar, P. G. Mickelson, M. Yan, B. J. DeSalvo, S. B. Nagel, and T. C. Killian, Phys. Rev. Lett. 103, 200402 (2009).

[35] S. Stellmer, M. K. Tey, R. Grimm, and F. Schreck, Phys. Rev. A 82, 041602 (2010).

[36] P. G. Mickelson, Y. N. Martinez de Escobar, M. Yan, B. J. DeSalvo, and T. C. Killian, Phys. Rev. A 81, 051601 (2010).

[37] S. Stellmer, B. Pasquiou, R. Grimm, and F. Schreck, Phys. Rev. Lett. 110, 263003 (2013).

[38] B. J. DeSalvo, M. Yan, P. G. Mickelson, Y. N. Martinez de Escobar, and T. C. Killian, Phys. Rev. Lett. 105, 030402 (2010).

[39] S. Stellmer, R. Grimm, and F. Schreck, Phys. Rev. A 87, 013611 (2013).

[40] G. Reinaudi, C. B. Osborn, M. McDonald, S. Kotochigova, and T. Zelevinsky, Phys. Rev. Lett. 109, 115303 (2012).

[41] M. Tomza, F. Pawlowski, M. Jeziorska, C. P. Koch, and R. Moszynski, Phys. Chem. Chem. Phys. 13, 18893 (2011).

[42] B. Pasquiou, A. Bayerle, S. M. Tzanova, S. Stellmer, J. Szczepkowski, M. Parigger, R. Grimm, and F. Schreck, Phys. Rev. A 88, 023601 (2013).

[43] B. H. McGuyer, M. McDonald, G. Z. Iwata, M. G. Tarallo, W. Skomorowski, R. Moszyński, and T. Zelevinsky, arXiv:1407.4752.

[44] W. Skomorowski, F. Pawłowski, C. P. Koch, and R. Moszynski, J. Chem. Phys. 136, 194306 (2012).

[45] S. B. Nagel, C. E. Simien, S. Laha, P. Gupta, V. S. Ashoka, and T. C. Killian, Phys. Rev. A 67, 011401 (2003).

[46] M. Yan, R. Chakraborty, A. Mazurenko, P. G. Mickelson, Y. N. Martinez de Escobar, B. J. DeSalvo, and T. C. Killian, Phys. Rev. A 83, 032705 (2011).

[47] F. H. Mies, W. J. Stevens, and M. Krauss, J. Mol. Spectrosc. 72, 303 (1978).

[48] K. T. Tang and J. P. Toennies, J. Chem. Phys. 80, 3726 (1984).

[49] G. W. King and J. H. van Vleck, Phys. Rev. 55, 1165 (1939).

[50] E. Tiesinga, C. J. Williams, and P. S. Julienne, Phys. Rev. A 57, 4257 (1998).

[51] J. Mitroy and J. Y. Zhang, Mol. Phys. 108, 1999 (2010).

[52] S. G. Porsev, M. S. Safronova, and C. W. Clark, Phys. Rev. A (to be published).

[53] M. S. Safronova, S. G. Porsev, U. I. Safronova, M. G. Kozlov, and C. W. Clark, Phys. Rev. A 87, 012509 (2013).

[54] S. Stellmer (private communication).

[55] S. Stellmer, F. Schreck, and T. Killian, in Annual Review of Cold Atoms and Molecules, Vol. 2, edited by K. W. Madison, Y. Wang, A. M. Rey, and K. Bongs (World Scientific, Singapore, 2014), Chap. 1. 
[56] P. S. Julienne, J. Res. Natl. Inst. Stand. Technol. 101, 487 (1996).

[57] G. F. Gribakin and V. V. Flambaum, Phys. Rev. A 48, 546 (1993).

[58] R. J. LeRoy and R. B. Bernstein, J. Chem. Phys. 52, 3869 (1970).

[59] M. Borkowski, P. S. Żuchowski, R. Ciuryło, P. S. Julienne, D. Kędziera, L. Mentel, P. Tecmer, F. Münchow, C. Bruni, and A. Görlitz, Phys. Rev. A 88, 052708 (2013).
[60] M. Kitagawa, K. Enomoto, K. Kasa, Y. Takahashi, R. Ciuryło, P. Naidon, and P. S. Julienne, Phys. Rev. A 77, 012719 (2008).

[61] D. DeMille, S. Sainis, J. Sage, T. Bergeman, S. Kotochigova, and E. Tiesinga, Phys. Rev. Lett. 100, 043202 (2008).

[62] O. Allard, S. Falke, A. Pashov, O. Dulieu, H. Knöckel, and E. Tiemann, Eur. Phys. J. D 35, 483 (2005).

[63] M. Yan, Ph.D. thesis, Rice University, 2013. 